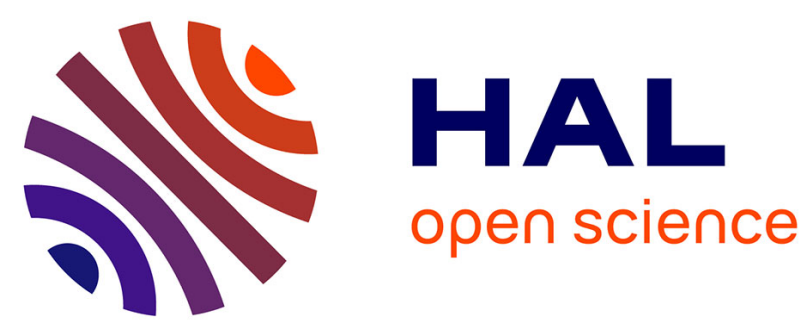

\title{
Insights into the $\pi-\pi$ interaction driven non-covalent functionalization of carbon nanotubes of various diameters by conjugated fluorene and carbazole copolymers
}

\author{
Robert Benda, Gaël Zucchi, Eric Cancès, Bérengère Lebental
}

\section{To cite this version:}

Robert Benda, Gaël Zucchi, Eric Cancès, Bérengère Lebental. Insights into the $\pi$ - $\pi$ interaction driven non-covalent functionalization of carbon nanotubes of various diameters by conjugated fluorene and carbazole copolymers. Journal of Chemical Physics, 2020, 152 (6), pp.064708. 10.1063/1.5133634 . hal-02502707

\section{HAL Id: hal-02502707 \\ https://hal.science/hal-02502707}

Submitted on 1 Dec 2020

HAL is a multi-disciplinary open access archive for the deposit and dissemination of scientific research documents, whether they are published or not. The documents may come from teaching and research institutions in France or abroad, or from public or private research centers.
L'archive ouverte pluridisciplinaire $\mathbf{H A L}$, est destinée au dépôt et à la diffusion de documents scientifiques de niveau recherche, publiés ou non, émanant des établissements d'enseignement et de recherche français ou étrangers, des laboratoires publics ou privés. 


\title{
Insights into the $\pi-\pi$ interaction driven non-covalent functionalization of carbon nanotubes of various diameters by conjugated fluorene and carbazole copolymers
}

\author{
Robert Benda, ${ }^{1,2}$, a) Gaël Zucchi, ${ }^{1}$ Eric Cancès, ${ }^{2}$ and Bérengère Lebental ${ }^{1,3}$ \\ 1) LPICM, CNRS, Ecole Polytechnique, Institut Polytechnique de Paris, Route De Saclay, 91128 Palaiseau, \\ France \\ ${ }^{2)}$ CERMICS, Ecole des Ponts and INRIA, Université Paris-Est, 6-8 avenue Blaise Pascal 77455 Marne-la-Vallée, \\ France \\ 3) Université Paris-Est, IFSTTAR, 14-20, Boulevard Newton, 77420 Champs-sur-Marne, \\ France
}

We investigate the interaction of polyfluorene and fluorene/carbazole copolymers bearing various functional groups and side chains with small to large diameter - from 1.7 to $9 \mathrm{~nm}$ - carbon nanotubes (CNTs) in vacuo. We use variable-charge molecular dynamics simulations based on the reactive force field ReaxFF. We show that non covalent functionalization of nanotubes, driven by $\pi-\pi$ interactions, is effective for all the polymers studied, thanks to their conjugated backbone and regardless of the presence of specific functional groups. The geometry at equilibrium of these polymer/CNT hybrids is analyzed in detail at the scale of each fluorene or carbazole unit. The role of both the functional groups and the alkyl chains length is analyzed in detail. Adsorption of the polymers on the nanotube sidewalls is shown to be either complete - with the whole chain physisorbed - or partial - due to intrachain coiling or interchain repulsion - depending on the initial geometry, number of polymers and nanotube diameter. Energetic arguments supplement the described geometric features. Both energetic and geometric adsorption features are derived here for the first time for large diameter carbon nanotubes (up to $9 \mathrm{~nm}$ ) and fluorene/carbazole copolymers having up to 30 monomers and bearing different functional groups. The force field ReaxFF and its available parametrization used for the simulations are validated thanks to a benchmark and review on higher level quantum calculations - for simple $\pi-\pi$ interacting compounds made up of polycyclic aromatic molecules adsorbed on a graphene sheet, or bilayer graphene. Although it is shown that the influence of the nanotube chirality on the adsorption pattern and binding strength can not be discussed with our method, we highlight that an available force field such as ReaxFF and its parametrization can be transferable to simulate new systems without specific re-parametrization, provided that this model is validated against reference methods or data. This methodology proves to be a valuable tool for optimal polymer design for nanotube functionalization at no re-parametrization cost, and could be adapted to simulate and assist the design of other types of molecular systems.

\section{INTRODUCTION}

Given their peculiar electronic properties and their sensitivity to small geometric or environmental changes, carbon nanotubes (CNTs) have already found a wide range of applications, for instance as active material for sensors directly embedded in concrete, to detect strains and anticipate structural failings in buildings. ${ }^{1}$ They have also been used as active material for thermoelectric sensors, ${ }^{2}$ biochemical sensors, ${ }^{3}$ humidity sensors, ${ }^{4,5}$ and chemical sensors, ${ }^{6-9}$ in particular for gas sensing ${ }^{10,11}$ or pH measurement. ${ }^{12}$

Functionalization of CNTs by polymers has also been investigated for various applications, as reviewed e.g. in Refs. ${ }^{14}$ This requires dispersion of the CNTs prior to functionalization by the polymers. Nanotubes tend indeed to aggregate and form bundles, because of strong $\pi-\pi$ stacking interactions ${ }^{15}$ between their sidewalls.

\footnotetext{
a)Electronic mail: robert.benda@ polytechnique.org
}

This is a feature even stronger for Multi-Walled CNTs (MWNTs) than Single-Walled CNTs (SWNTs) due to their lower curvature and larger possible contact surface. Improving the dispersion of CNTs in solution has thus been a main topic of research, either via covalent ${ }^{16}$ or non-covalent functionalization. ${ }^{17-19}$ The second main objective of CNT/polymer functionalization is CNTs sorting, by selectively functionalizing (and consequently, dispersing) SWNTs, depending on their diameter or chirality, ${ }^{17,20,21}$ enabling for instance to target semi-conducting nanotubes for Field-Effect transistor purposes. $^{22,23}$ These chirality selective purposes have led to numerous classical molecular dynamics studies of polymer/CNT compounds, ${ }^{24-26}$ notably to understand selective dispersion of nanotubes by polymers and the main geometrical features of the physisorption at the interface - down to the atomic scale. Such simulations were also used to estimate the typical binding energies and confirm the tendencies of specific polymers to interact preferentially with specific nanotubes (chirality 'map') that were experimentally observed. ${ }^{27-29}$ Other purposes of CNTs functionalization are the use of 
CNT/polymer hybrids for sensing, ${ }^{30-32}$ as reinforcement fibers, ${ }^{33-35}$ and for photovoltaic applications. ${ }^{23,36-39}$ Charge transfer at the CNT/polymer interface has also been studied, e.g. to estimate the exciton binding energy and predict which type of chirality would be best suited for photovoltaic heterojunction devices. ${ }^{39,40}$ Non-covalent functionalization of CNTs by polymers which can be electron donors or acceptors - is indeed a promising idea to create $n-p$ junctions and avoid electron-hole recombination, as CNT and polymer are physically separated.

Functionalizing CNTs with conjugated polymers for sensor applications has appeared in the field of gas sensing, ${ }^{14,41}$ drug delivery, ${ }^{42}$ chemical sensing $9,31,32,43,44$ or biosensing. ${ }^{45,46}$ If many articles have adressed the question of selective non-covalent functionalization of SWNTs (with diameter of order 0.7 to $1.5 \mathrm{~nm}$ ) by conjugated polymers, and in particular polyfluorene derivatives, ${ }^{28,29,47}$ the features of MWNTs (or very large diameter CNTs) and polyfluorene hybrids have not been investigated yet by simulation techniques. Conjugated polymers such as polyfluorene copolymers have a conjugated backbone and can interact favorably with the CNT sidewalls through $\pi-\pi$ interactions. They are thus good candidates for CNTs non-covalent functionalization.

The total binding energy of conjugated polyfluorene polymers on CNTs increases with increasing (Single Walled) nanotube diameter according to Nish et al. ${ }^{25}$ and Salazar-Rios et al. ${ }^{47}$ for small diameter - from 1 to $1.5 \mathrm{~nm}$ - carbon nanotubes and the same type of polymers. Salazar-Rios et al. also analyzed the influence of the polymer flexibility and reported that the more flexible the backbone, the easier the helical wrapping. They also compared the cost in torsional energy due to wrapping to the adsorption binding energy, and the influence of the length of alkyl chains - the longer, the stronger the adsorption. ${ }^{47}$

Yet, the physics of the interaction between conjugated polymers and Multi Walled CNTs might be somewhat different as the latter have several inner shells, which impact the mechanical properties - e.g. the buckling behavior under compression, studied e.g. in Refs. ${ }^{33,35}$ at the light of the cohesive vdW forces between the layers - and much larger diameters, usually ranging from $10 \mathrm{~nm}$ to $20 \mathrm{~nm}$, hence lower surface curvatures. In Ref., ${ }^{34} \mathrm{Li}$ et al. concluded that the total binding energy of acrylonitrile-butadiene rubber (aliphatic) polymer chains increases with the number of walls of the underlying nanotube. Boon and co-workers studied poly(3-hexylthiophene-2,5-diyl) (P3HT) interacting with CNTs of diameters ranging from 1.4 to $5.4 \mathrm{~nm},{ }^{26}$ concluding that the interaction energy varies slightly with increasing CNT diameter.

In this article, we probe for the first time the interac- tion between large diameter (up to $9 \mathrm{~nm}$, representative of a MWNT outer shell) CNTs, as well as Double Walled CNTs (DWNTs), and one or several fluorene/carbazole copolymers. These polymers bear functional groups designed for sensing applications. We also perform simulations for a smaller diameter $(1.7 \mathrm{~nm}) \mathrm{CNT}$ and compare the geometric and energetic features over the whole diameter range. Variable-charge molecular dynamics, based on ReaxFF force field, is used. Structural features of the physisorption of conjugated polymers on CNTs are analyzed in detail, at the scale of each monomer, for the first time. The local stable geometries of the fluorene groups, when adsorbed on the CNT surface (either in a parallel or perpendicular way), are analyzed depending on the alkyl chains length, or on the functional groups their carry. A comparison of the relative binding energies of the different monomers, depending on their specific adsorbed conformations, is provided. A qualitative analysis of polymer coiling, intra and inter chain steric repulsion and their impact on the adsorption is performed. Morever, we perform a benchmark in the Supporting Information (SI) to show that the force field ReaxFF, and one of its available parametrizations chosen here, are transferable to our nanotube/polymer compounds to a satisfactory extent, allowing to discuss the main structural and energetical features of nanotube non-covalent functionalization by conjugated polymers. This methodology could prove useful to simulate a wide range of new systems with already available force fields, without any new parametrization work. This SI is based on Refs. ${ }^{48-73}$

\section{METHODS}

\section{Simulation technique :}

Non-covalent functionalization of CNTs by conjugated polymers is likely to involve subtle charge effects, partly due to $\pi-\pi$ interactions. ${ }^{15}$ The latter are indeed mainly due to van der Waals interactions, but also dependent on stabilization effects due to electron delocalization. ${ }^{15,60,64}$ Looking for the best approach to capture this effect, the compromise between accuracy and computational effort arises. At the scale of a single, $20 \mathrm{~nm}$ long large-diameter (e.g. at least 4nm) CNT and a polymer made up of 30 monomers, which can be deemed the simplest possible model of a CNT-polymer compound, the system is already made up of a few tens of thousand of atoms. This impedes the use of quantum chemistry or density functional calculations for dynamical simulation purposes. Moreover, many classical force fields, such as CHARMM, ${ }^{74}$ GROMOS, ${ }^{75}$ AMBER, ${ }^{76,77}$ are mainly targeted at the simulation of biological systems, composed of well known and accurately parametrized building blocks. Yet, some classical force fields have been parameterized to simulate molecular systems similar (but not rigorously equivalent) to our 
compounds of interest, such as AMBER, ${ }^{76,77}$ MM3, ${ }^{78}$ PCFF $^{79,80}$ and COMPASS ${ }^{81}$ (the two latter being class II force fields ${ }^{82}$ and using bond increments to compute atomic partial charges). The MM3 force field has been used to simulate polyfluorene derivatives-SWNT compounds, ${ }^{18,25}$ as well as the generalized AMBER force field ${ }^{83}$ in Ref. ${ }^{27}$ and the CVFF (class I) force field (see e.g. Refs. ${ }^{84}$ or Appendix of Ref. ${ }^{85}$ for parameters) in Refs. ${ }^{28,29}$ both in the case of polyfluorene derivativesSWNTs compounds. The PCFF force field has been used to simulate the interaction of thiophene-containing conjugated polymers with SWNTs, ${ }^{86}$ while the AMBER force field ${ }^{77}$ has been used e.g. to probe DNA-SWNT interaction in explicit water solvent. ${ }^{45}$ The COMPASS force field, initially developed for common small organic molecules and polymers, has also been used to simulate carbon nanotubes and polymer hybrids. ${ }^{34}$ Finally, the INTERFACE force field, ${ }^{87}$ which was built as an extension of previous force fields such as CVFF, PCFF or CHARMM for inorganic materials, has also recently been used to simulate similar systems. ${ }^{19}$ Nevertheless, because of the specific new functional groups they carry, our fluorene and carbazole copolymers of interest (see next section, P1 and P2 polymers) have never been wholly parametrized for any of the previously mentioned classical force fields. Simulating a new, non-biological molecular system (such as our new functional groups grafted to the conjugated polymer backbones, see below) with such a force field implies much effort to derive a consistent parametrization (bonded parameters, partial charges, etc.) for the new atomic environments, whose validation is very demanding, and which is generally poorly transferable to different chemical contexts. With such classical force fields, partial charges are assigned to the atoms of the system once and for all, and kept fixed all over the simulation, which prevents to capture any charge redistribution effects.

Let us point that generic force fields, such as the Universal force field ${ }^{88}$ or the DREIDING force field, ${ }^{89}$ were explicitly designed so that any new molecule is easily parametrized. Indeed, all the parameters of these force fields (for different combinations of atoms in various environments) are derived thanks to very simple combination rules, from atomic constants only for UFF, ${ }^{88}$ or from very simple hybrization considerations in DREIDING. ${ }^{89}$ This DREIDING force field has been been used several times to simulate polymer nanotube non-covalent interactions (both for aliphatic or conjugated chains), e.g. in Refs. ${ }^{90,91}$ UFF was also used e.g. in Ref. ${ }^{24}$ (as a preparation to DFT optimizations) or in Ref. ${ }^{92}$ to simulate fluorene/thiophene copolymers interacting with small diameter $(7,5)$ SWNTs. This relatively simple parametrization procedure for UFF or DREIDING may turn out to play at the expense of results accuracy. Indeed, although the force field parameters for a new molecule are easily obtained, there is no guarantee that such simple and general parametrization rules provide sufficient transferability of these force fields from one compound to another. Moreover, such generic force fields are not focused on the electrostatics term - which is sometimes even omitted in $\operatorname{UFF}^{88}$ (e.g. if atomic partial charges are not available or validated) - and the role of electrostatics can rather be implicitly contained in the valence parameters.

Due to the points previously detailed, we decided to use variable-charge molecular dynamics in this paper, with no need to initially parametrize partial charges. Such a technique (that we deem more accurate in terms of electrostatics description, compared to the methods used by the previously mentioned fixed-charge force fields) relies on geometry-dependent charge calculations - as introduced in Ref. ${ }^{93}$ This method enables to recompute partial charges on atoms dynamically, at each step, following the geometry evolution of the system - by minimizing the total electrostatic energy i.e. solving a linear system of $N$ (the number of atoms) equations with the atomic partial charges as unknowns. The charge equilibration scheme Qeq ${ }^{94}$ is used here to compute the partial charges of the polymer or organic molecule, and of the nanotube, at each step of the molecular dynamics (MD) simulation or structural minimizations. The Qeq model has some flaws, reported in Ref. ${ }^{95}$ For instance, it does not behave correctly (i.e. does not yield correct partial charges) when bonds are broken, but here it is used in a chemical context devoid of any bond dissociation. However, the overestimation of charges for weakly interacting, widely separated systems ${ }^{95}$ has to be kept in mind as we are presently dealing with non-covalent functionalization i.e. polymers and nanotube separated by distances of at least $3.4 \AA$ (see section Results).

Solving the Qeq linear system at each step leads to a computational cost about ten times larger than for classical force fields but still allows to study systems made up of tens of thousand of atoms over reasonable time scales. The reactive force field ReaxFF, first designed by van Duin et al. for hydrocarbons, ${ }^{96}$ was designed to include dynamic partial charges adjustment over the course of a simulation, and can be used in conjunction with the Qeq scheme. ReaxFF force field allows to perform molecular dynamics with the possibility of breaking and creating bonds during the course of the simulation, thanks to the concept of bond order, integrated into the functional form of the force field. ${ }^{96}$ It has enabled realistic simulations of numerous phenomena involving chemical dissociation events, among them catalysis ${ }^{97}$ or amine molecules dissociation. ${ }^{48}$ MD simulations with ReaxFF as underlying potential have been mostly used to study systems with frequent changes of connectivity, ${ }^{48}$ as ReaxFF was more chemistry-targeted. Still, ReaxFF can also be used in lower energy contexts (where the probability of bond breaking is very low), as in Ref., ${ }^{98}$ which studies the SWNT and polymer non-covalent 
interaction through ReaxFF-based MD, while no change in connectivity or bond breaking is expected. Only small diameter (around $1 \mathrm{~nm}$ ) nanotubes were adressed, with polythiophene polymers (among others) of only a few monomers.

In this work, we use the force field ReaxFF and the Qeq partial charge recomputation method ${ }^{94}$ to study the interaction - in vacuo - between small to large diameter (around $9 \mathrm{~nm}$ ) CNTs - the latter being representative of MWNTs outer shells - and polyfluorene derivatives. This is to our knowledge the first time this force field is used to simulate large diameter CNT and polymer compounds.

The package USER-REAXC, an implementation in C-language of ReaxFF ${ }^{99,100}$ embedded in LAMMPS code, ${ }^{101}$ was used for the simulations. The ReaxFF parameters taken for the simulations are those available from article, ${ }^{48}$ derived for systems containing $\mathrm{C}, \mathrm{H}, \mathrm{N}$ and $\mathrm{O}$ in a high energy context. Because this force field and the associated parametrization were not explicitly derived for the specific systems we study - i.e. to study possible adsorption geometries and energies of conjugated polymers on CNT surfaces -, we perform a benchmark of the force field ReaxFF with this particular parametrization. We study model systems, made up of polycyclic aromatic molecules adsorbed on a graphene surface, the results being reported in the Supporting Information. Adsorption geometries and energies are compared between ReaxFF, Lennard-Jones potential, and higher level ab-initio methods. The validity of the method is asserted, showing that the binding energy and distance are correctly reproduced by ReaxFF for $\pi-\pi$ stacking interacting systems. From a methodological point of view, one of the goals of the article and Supporting Information is also to test to which extent a given available (i.e. without the need to rederive specific parameters for each new system) force field (such as ReaxFF) is transferable to functionalized carbon nanotubes, by testing it and performing a benchmark against one or several quantum reference methods. Given the need to simulate a wide class of different systems or polymers to orientate device fabrication and to provide useful insight to experimentalists manipulating these systems, rederiving a specific parametrization for each (non-transferable to other systems and other contexts) would prove too long.

The system at stake is not periodized, but simply included in a sufficiently large simulation box. For the MD simulations, we use a Nose Hoover deterministic thermostat as a sampling procedure to reproduce the statistics of the NVT canonical ensemble, with a $300 \mathrm{~K}$ target temperature and time steps of $0.3 \mathrm{fs}$. A velocity Verlet scheme is used to integrate the corresponding equations of motion. Stable and metastable configura- tions obtained during the MD phase space exploration are extracted and their geometry separately further minimized (at zero temperature) to deduce adsorption energies. On average, simulations of 150,000 to 300,000 steps (depending on the polymer length, average initial distance and configuration of the polymer chain with respect to the nanotube surface) of $0.3 \mathrm{fs}$, i.e. $45 \mathrm{ps}$ to $90 \mathrm{ps}$, were performed. This duration was indeed long enough for polymers to adsorb and stabilize, as well as for all terms contributing to the total energy to become constant. Twice more number of steps were roughly needed to achieve convergence for twice longer polymer chains of similar initial configuration with respect to the nanotube. Although bond dissociation events are possible with such a reactive force field, we did not observe any bond breaking for these simulations in the NVT ensemble, at $300 \mathrm{~K}$, when the system was initially energy minimized at $0 \mathrm{~K}$, and using a small enough timestep (smaller than 0.3 fs to ensure numerical stability).

\section{Systems studied :}

The polymers chosen to probe the interaction with CNTs are polyfluorene derivatives or fluorene/carbazole copolymers (including some carbazole groups replacing periodically fluorene monomers). These polymers have been patented recently ${ }^{31}$ and are related to undergoing experimental works on chemical sensors. The backbones of these polymers are made up of alternating fluorene di-hexyl and carbazole units. They can be (in the case of copolymers) separated periodically by single units bearing grafted functional groups ${ }^{31}$ - known to complex specific ions, also called 'probes' in the following which can be attached either to carbazole or fluorene groups. The polymer may also be devoid of any probe. It is the case of polyfluorene homopolymers, which are used as benchmarks in the following, to isolate the geometrical and energetic effects of the probes carried by their counterparts.

The CNTs used in the simulations are finite portions of infinite tubes (of $20 \mathrm{~nm}$ long). They are not periodized but are hydrogenated at their extremities to avoid edge effects due to unsaturated carbon atoms. They are initially energy-minimized to relax the forces due to this artificial nanotube shortening. All polymers and CNTs are initially built and visualized thanks to SAMSON software $^{102}$ (as well as the simulation output geometries).

As a benchmark, we first studied the interaction between a $4 \mathrm{~nm}$ diameter armchair nanotube (portion of about $20 \mathrm{~nm}$ long) and poly(9,9-dihexylfluorene) polymers made up of 15 and 30 monomers. This value of $4 \mathrm{~nm}$ diameter was chosen about twice smaller than the outer shell of the MWNTs diameter (about $9.5 \mathrm{~nm}$ according to the supplier - Nanocyl NC 3100 datasheet) to reduce computational expenses. Increasing the diame- 
ter of the CNT from $4 \mathrm{~nm}$ to $5 \mathrm{~nm}$ adds around 2700 new carbon atoms for a total length of the nanotube of $20 \mathrm{~nm}$, which results in a notably higher computational cost.

We then compared the interaction of one or several fluorene:carbazole copolymers (denoted as P1 in the following) made up of 10 and 30 monomers, with 1.7 $\mathrm{nm}, 9 \mathrm{~nm}$ diameter (armchair) nanotubes, and a Double Walled nanotube of $9 \mathrm{~nm}$ diameter (armchair) outer shell. The length of the simulated P1 copolymers - designed to interact with heavy metal ions and whose structure is displayed in figure 1 - is chosen to match experimental data, ${ }^{103}$ as well the 1:9 proportion of carbazole units bearing functional groups relatively to fluorene groups bearing two hexyl chains ${ }^{103}$ - responsible for the solubility. Similarly, we studied the interaction with a large diameter ( $9 \mathrm{~nm}$ diameter) nanotube of a polyfluorene polymer with one every ten fluorene group bearing a bis urea group (in the same 1:9 proportion), denoted as P2 in the following (its structure is displayed in figure 2).

Some examples of CNT-polymer compounds systems initial geometries for MD simulations are displayed in figures 3, 7 and 9. They are chosen so that polymer chains are far enough from the CNT axis, and not parallel to it, to avoid favoring any specific adsorption mode. The smallest distance between the polymer backbone and the CNT is always of order or larger than $10 \AA$, to avoid any initial interaction between the polymer and the CNT which could bias the resulting exploration. Indeed, the cutoff distance for non-bonded - van der Waals and Coulomb - interactions is $10 \AA$ in ReaxFF (Taper correction ${ }^{104}$ ), so that polymer atoms initially do not interact with CNT atoms. Varying qualitatively the initial geometries (i.e. the relative position of the polymer with respect to the CNT), we were able to highlight the influence of the starting configuration as regards the possible intrachain coiling of the polymer (or interchain steric repulsion for two polymers) in the final adsorbed geometry (as discussed in the Results section).

\section{RESULTS AND DISCUSSION}

Comparing the interaction of a CNT with polyfluorene homopolymers, on the one hand, and on the other hand with fluorene:carbazole copolymers, bearing not only alkyl chains, but also chemical groups - designed to

The general trend observed during the molecular dynamics explorations is the following. The conjugated polymers first explore their own configurational space,
Before bringing together the CNT and the polymer, both are separately relaxed within LAMMPS, with the same force field ReaxFF. Geometric and energetic features derived from all these simulations are discussed in the next part.

The influence of the chirality of the CNT has not been investigated in this study. All investigated CNTs are thus armchair. Indeed, we show in the Supporting Information that although the most stable binding geometry $(A B)$ and binding energy - for aromatic systems stacked on graphene - are correctly recovered by ReaxFF, the influence of the nanotube chirality on the adsorption pattern and binding strength of conjugated polymers can not be discussed with ReaxFF. The energy barrier for the displacement of an aromatic polycyclic molecule parallel to the graphene sheet - e.g. between two extreme adsorption modes $\mathrm{AB}$ and $\mathrm{AA}$ - is indeed found to be underestimated with ReaxFF by one order of magnitude, compared to the best performing DFT-D methods (e.g. $\omega \mathrm{B} 97 \mathrm{X}-\mathrm{D}$ functional and at least $6-31 \mathrm{G}^{*}$ basis set) for these weakly interacting systems. Simple semi-empirical force fields do not satisfactorily reproduce the barrier derived by DFT (or higher-level quantum mechanical calculations) on similar systems. Thus, the precise relative orientation of the polymer chain adsorbed on (i.e. functionalizing) the tube surface can not be discussed. Yet, such force fields - and in particular ReaxFF, which does no imply any re-parametrization effort - are the only possible tool at this system size, to explore the configurational space of CNT-polymer compounds at reasonable cost. Although they appear not to include enough physical ingredients to capture unambiguously the corrugation of the translational energy landscape, the main adsorption features - as the perpendicular and parallel geometry for the adsorbed fluorene groups, or the coiling patterns (see below) - can be analyzed correctly. The adsorption distance and the magnitude of the binding energy are indeed shown to be well captured in the Supporting Information.

complex specific ions in water ${ }^{31}$ - grafted periodically to either fluorene or carbazole groups, allows to isolate the role of these probes on the non-covalent functionalization.

thanks to the free rotations (at $300 \mathrm{~K}$ ) along single bonds linking successive monomers, without yet undergoing any attraction of the nanotube. The beginning of the MD 


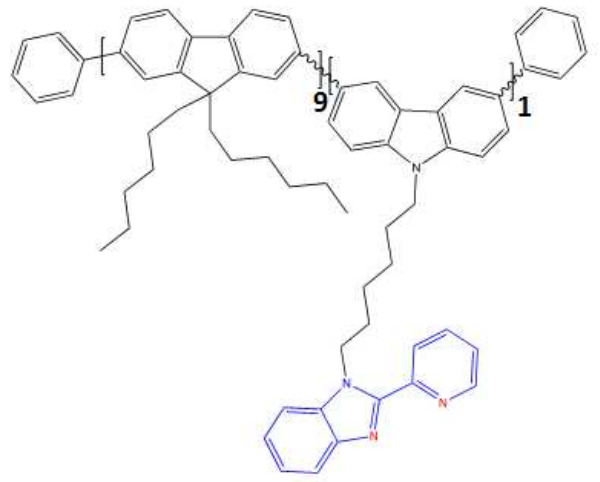

FIG. 1: Structure of P1 carbazole:fluorene copolymer

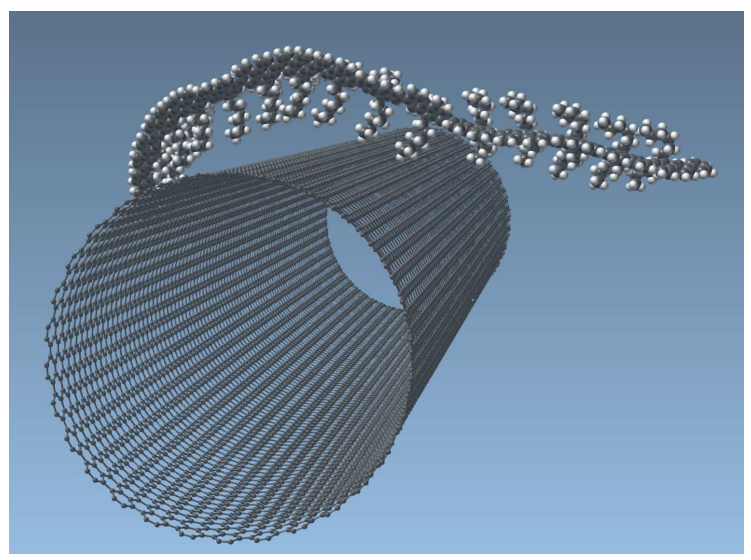

FIG. 3: Initial geometry of the 15-monomers long poly(9,9-dihexylfluorene) and neighboring $4 \mathrm{~nm}$ diameter and $20 \mathrm{~nm}$ long CNT. Hydrogen atoms are displayed in white, and carbon atoms in grey.

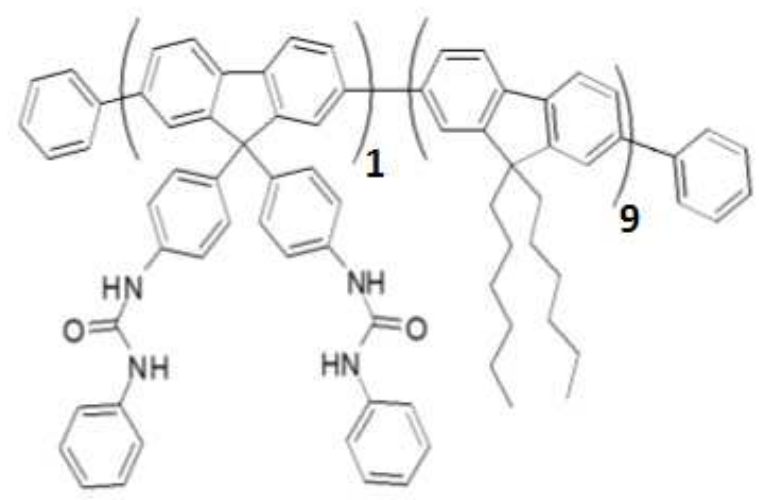

FIG. 2: Structure of P2 polyfluorene polymer. ${ }^{31}$

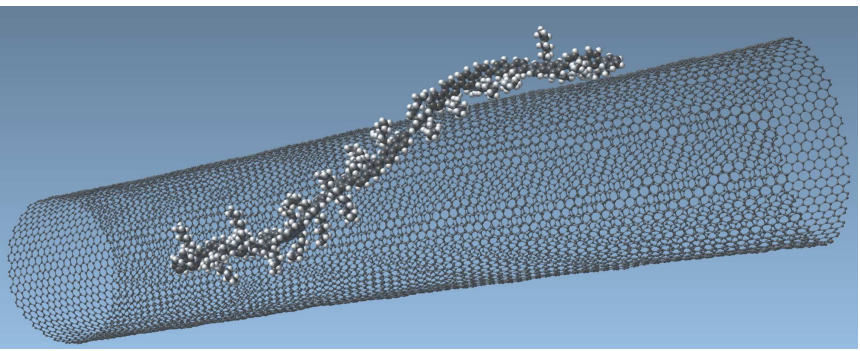

FIG. 4: 15-monomers long poly(9,9-dihexylfluorene) polymer adsorbed on a $4 \mathrm{~nm}$ diameter and $20 \mathrm{~nm}$ long CNT, configuration extracted after 150,000 steps of $0.3 \mathrm{fs}$ i.e. $45 \mathrm{ps}$ (from starting geometry 3 ) and further minimized.

physisorbed, in the particular (but very likely) case of a coiled chain (see for instance the possible adsorption geometries displayed in figures 12, 13 and 18). This feature is due to the random space exploration of the chain prior to the adsorption on the CNT surface. The polymer, if initially far enough from the CNT surface, is long enough to make 'loops' around itself. The part of the chain that is adsorbed can prevent other monomers (further away along the chain), located on top of it, to adsorb as well - simply because of steric hindrance. Such intrachain coiling has been reported (thanks to molecular dynamics NVT simulations) for flexible backbone polymers adsorbed on a $0.8 \mathrm{~nm}$ diameter and $12.5 \mathrm{~nm}$ long SWNT. ${ }^{91}$ The same authors reported that most polymers with stiffer backbones tend to remain more linear (with fewer, or without any intrachain coiling and loops) prior to adsorption on the this feature is likely to be conserved upon adsortion. Alternatively, the polymer can end up being only partly 


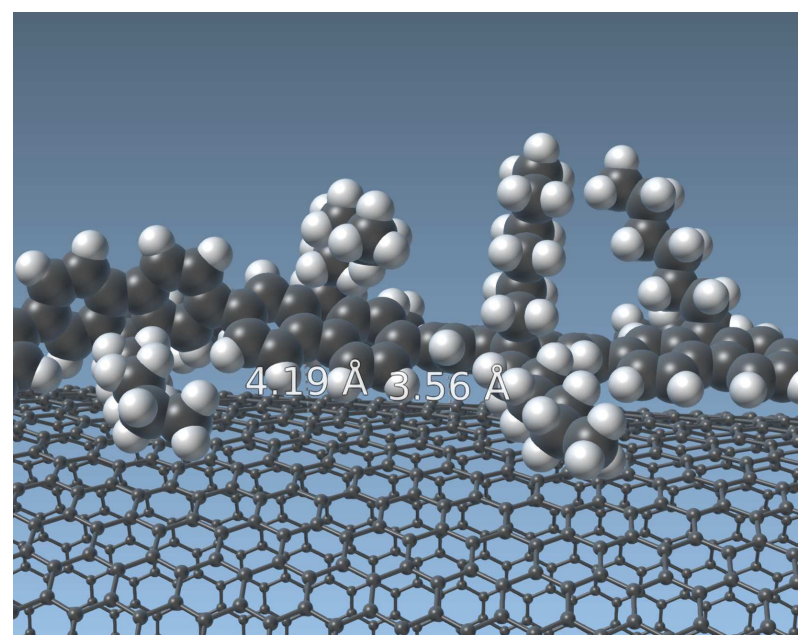

FIG. 5: Local geometry of neighboring fluorene groups parallel (right groups) and perpendicular (left group) to the $4 \mathrm{~nm}$ diameter CNT surface (with two indicative distances from the polymer backbone to the CNT surface) : the two possible local geometries observed in all adsorption geometries of polyfluorene polymers.

same SWNT, ${ }^{90}$ though it may be linked to the choice of starting configurations. Yet, for a poly(pyrrole) polymer made up of 30 units, an adsorption pattern with partial intrachain coiling and partial nanotube wrapping was reported, ${ }^{90}$ consistently with our following qualitative analysis (see next subsection). This departure of part of the polymer backbone from the CNT sidewalls has also been mentioned in Ref. ${ }^{29}$

- the fluorene group is perpendicular to the CNT surface (see figure 5, top left monomer or figure 6, two upper left groups), the carbon atom of the fluorene bearing the two hexyl chains being the closest to the CNT - at about $4 \AA$ of the surface. The two hexyl chains are adsorbed on the CNT and maximize their extension along the surface underneath, suggesting van der Waals interactions with the honeycomb lattice, already reported in Ref. ${ }^{105}$ for alkanes on graphite. The mean distance be-

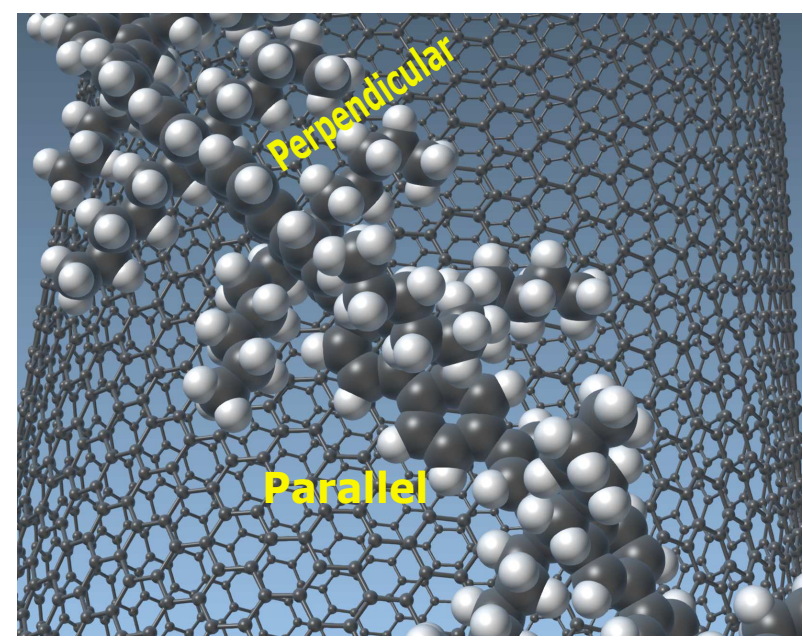

FIG. 6: Top view of two neighboring fluorene groups perpendicular (left upper groups) and parallel (right lower groups) to the $4 \mathrm{~nm}$ diameter CNT surface in the same adsorption geometry.

\section{A. Geometrical features}

Once the most stable configuration for the polymer on the CNT surface has been reached, the atoms of the backbone nearest to the surface are located at a distance of 3.5 $\AA$ to $4 \AA$ from the CNT sidewalls, as shown in figure 5. In the particular simulation of a 15 monomer-long poly $(9,9$ dihexylfluorene), with initial geometry displayed in figure 3, and 'final' geometry - after 150,000 steps of $0.3 \mathrm{fs}$ i.e. $45 \mathrm{ps}$ - displayed in figure 4 , the interaction between the polymer chain and the $4 \mathrm{~nm}$ diameter CNT is complete, i.e. every fluorene unit ends up being in contact with the CNT surface. Two main types of local adsorbed geometries are observed, and these observations are common to all the polyfluorene polymers studied in this paper (either for the whole chain, when it is entirely adsorbed, or for the part of the chain which is adsorbed, in the case of a coiled, not wholly adsorbed chain) :

tween the axis of the hexyl chains, which are parallel to the CNT, and the surface, is about 3 to 3.4 A.

- the fluorene group is stacked parallel to the CNT surface at an average distance of about $3.5 \AA$,- see figure 5 - suggesting $\pi-\pi$ stacking interactions between the $\pi-\pi^{*}$ electron systems of the aromatic fluorene groups and the CNT honeycomb lattice. One hexyl chain points outwards and 


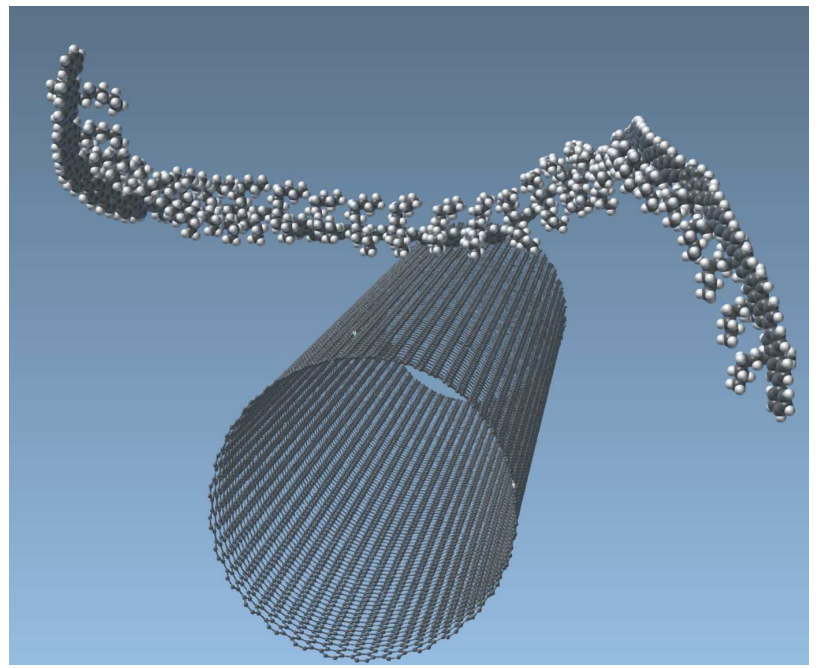

FIG. 7: Initial geometry of the 30-monomers long poly(9,9-dihexylfluorene) and neighboring $4 \mathrm{~nm}$ diameter CNT.

seems not to interact with the CNT, while the other one is parallel to the CNT surface, interacting through van der Waals dispersion forces with the underlying surface. The two hexyl chains thus fill the available space without steric repulsion and maximizing their interaction with the CNT surface. The stacking distance close to $3.4 \AA$ is consistent with the interlayer distance between two graphene sheets in graphite $^{64}$ and other physisorption equilibrium distances.

The existence of these two possible local geometries for fluorene groups has already been reported by Gomulya et $a .^{29}$ for similar polyfluorene derivatives, of varying alkyl chain length, functionalizing non-covalently SWNTs of around $1 \mathrm{~nm}$ diameter. For this small diameter range, a selectivity of the polymer adsorption with respect to the nanotube chirality was unveiled. The local geometries observed in both cases in Ref. ${ }^{29}$ - either parallel or perpendicular - are very similar to those found here for armchair nanotubes of diameter ranging from 1.7 to $9 \mathrm{~nm}$. Moreover, the adsorption of only one alkyl chain per fluorene unit parallel to the surface (the other alkyl chain pointing outwards) has also been recently mentioned for dodecyl chains. $^{47}$

At this stage, the question arises whether a specific local adsorption geometry of the fluorene group on the

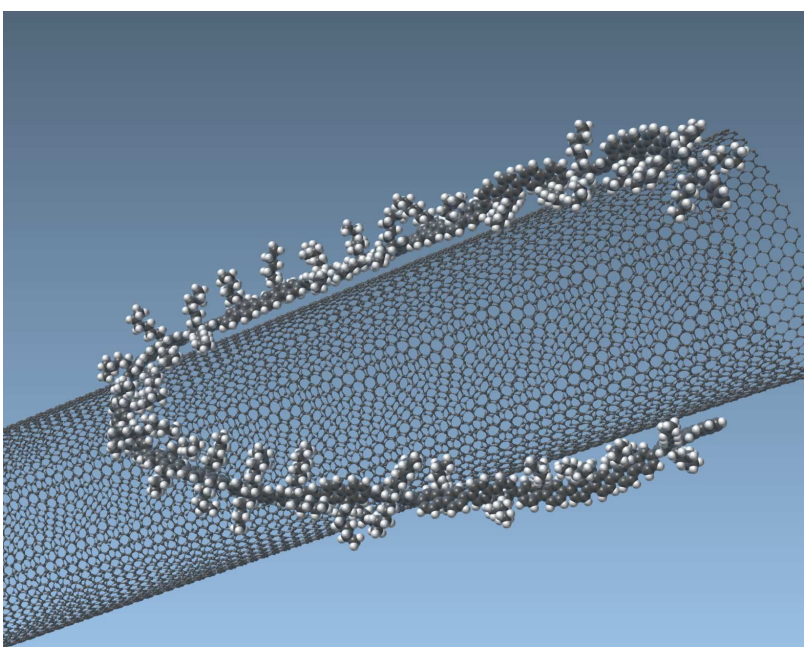

FIG. 8: Configuration of the 30-monomers long poly(9,9-dihexylfluorene) and $4 \mathrm{~nm}$ diameter CNT extracted after 250,000 steps of 0.3 fs i.e. 75 ps (from initial geometry displayed in figure 7) and further minimized.

Once the polymer has reached the most stable configuration on the CNT surface, it then remains physisorbed on the CNT surface all over the course of the configurational sampling. The polymer as a whole is seen to explore slightly different configurations on the surface. Its backbone is indeed able to deform on the surface, thanks to the energy provided by the heat bath at 300 $\mathrm{K}$. However, the fluorene groups always remain in the same local adsorbed perpendicular or parallel geometries defined above.

We obtained the same physisorption mechanism and local geometrical features - fluorene units being either perpendicular or parallel to the CNT sidewalls - for a twice longer (30 monomers, about $30 \mathrm{~nm}$ long) polyfluorene chain. Examples of initial geometry and of final adsorbed configuration are displayed in figures 7 and 8 respectively. The u-shaped geometry of the adsorbed 30 monomer-long polyfluorene is due to the specific initial configuration of the polymer backbone relatively to the nanotube chosen for this simulation. More generally, no preferential general adsorption pattern for the whole chain has been unveiled.

CNT surface (either perpendicular or parallel) is favored. At first sight, for the two poly(9,9-dihexylfluorene) 


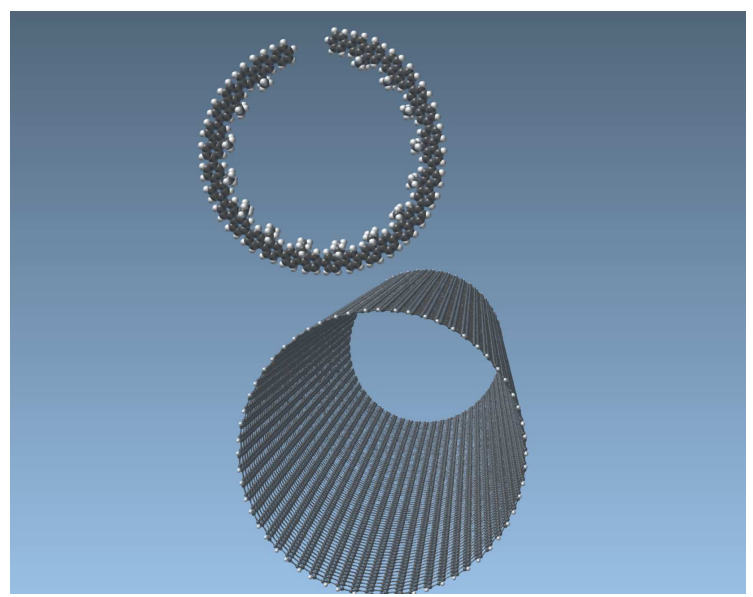

FIG. 9: Initial geometry of the 15-monomers long poly(9,9-dimethylfluorene) and neighboring $4 \mathrm{~nm}$ diameter, $20 \mathrm{~nm}$ long CNT.

polymers of different lengths studied, the ratio of perpendicular to parallel units seems to be close to unity whatever the nanotube diameter. Naturally, some variability occurs depending on the particular initial orientation of the polymer chain with respect to the CNT initially, and the direction of exploration followed by the sampling algorithm. The local geometry of each unit - either perpendicular or parallel - which is selected

\section{Influence of the length of the alkyl chains :}

To understand the influence of alkyl chains on the local adsorption geometries of the fluorene groups, we performed similar MD calculations (with the same 4 nm diameter CNT) with a poly(9,9-dimethylfluorene) polymer - also made up of 15 monomers, but simply replacing every hexyl chain with a methyl group. The initial geometry is displayed in figure 9 while the adsorbed S-shaped configuration, after interaction, is displayed in figure 10. Here, all the fluorene monomers unambiguously end up parallel to the CNT surface, even though the initial configuration was deliberately set with the polymer chain axis perpendicular to the CNT (which, at first sight, should rather lead to fluorene units adsorbed perpendicularly to the surface). This result suggests that the length of the alkyl chains grafted to the fluorene units is accountable for the appearance of local stable perpendicular geometries in the adsorbed configurations, which had not been reported yet. It is validated by energetic considerations hereafter.

Let us comment on this important feature. A fluorene unit bearing two hexyl chains - as for the poly(9,9-

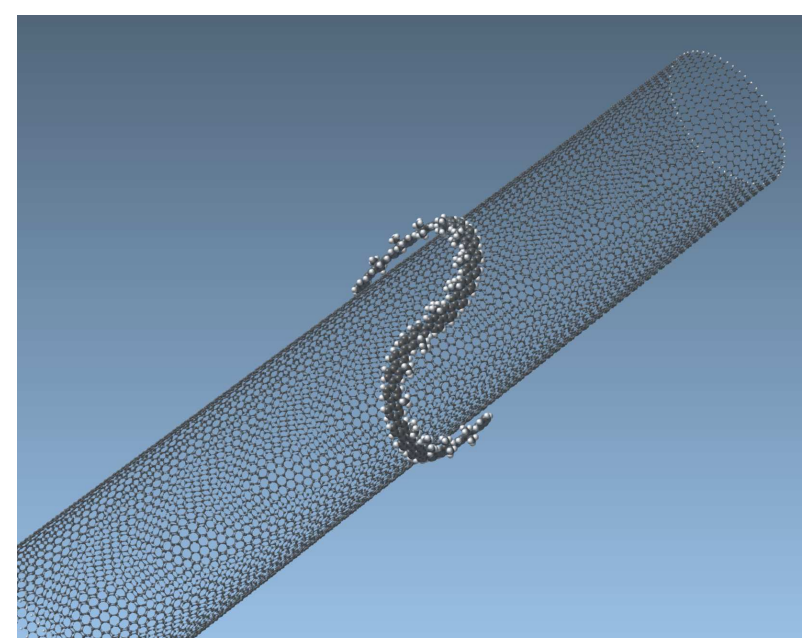

FIG. 10: Adsorbed configuration of the 15-monomers long poly(9,9-dimethylfluorene) on a $4 \mathrm{~nm}$ diameter, 20 $\mathrm{nm}$ long CNT (extracted after 60,000 MD steps of $0.3 \mathrm{fs}$ i.e. $18 \mathrm{ps}$, and further minimized).

when it approaches close enough to the sidewalls thus appears to depend on the initial distance between the polymer and the CNT, and on the dynamical evolution of inter-rings torsion angles between successive fluorene groups. It does not further evolve during the simulation once the polymer is physisorbed. This question will be further addressed in the energetic features section.

hexylfluorene) polymers studied -, once adsorbed on the CNT surface (as shown for instance in figures 5 and 6 ), cannot switch from the parallel to the perpendicular conformation because of the distance to the surface, which is too small to allow any rotation of the whole unit. On the contrary, methyl groups are small enough to allow such a rotation, simply because the size of a $\mathrm{CH}_{3}$ group is smaller than the distance of about $3.4 \AA$ to the surface underneath. For fluorene units bearing hexyl chains, steric (entropic) effects prevent the fluorene units from rotating over the CNT surface, once they are adsorbed.

These two benchmark simulations tend to suggest that the longer the alkyl chain, the more possible adsorbed configurations the polymer can adopt (i.e. the higher the configurational entropy). At least, when switching from methyl chains to hexyl chains attached to the fluorene units, perpendicular local geometries become possible, on top of parallel ones. This conclusion was also mentioned by Gomulya et al. for alkyl chains ranging from 6 to 18 carbon atoms. ${ }^{29}$ 


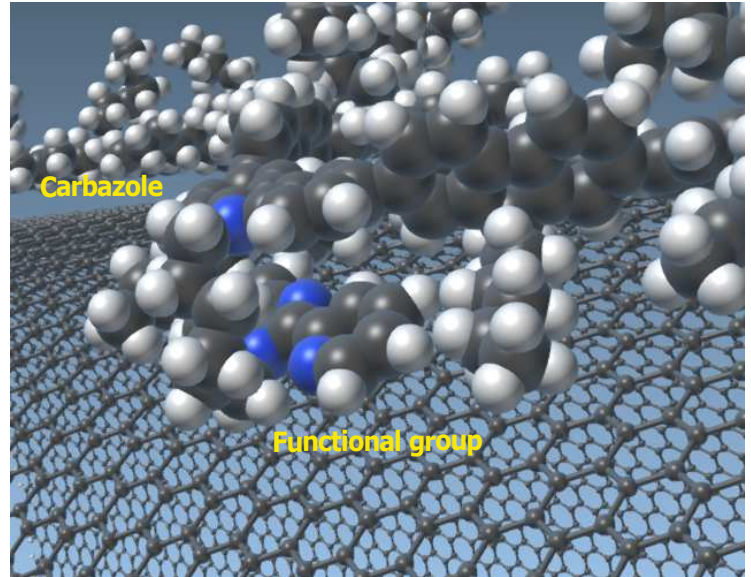

FIG. 11: Example of a carbazole group (belonging to a 30-monomers long $\mathrm{P} 1$ polymer) not adsorbed on the CNT surface ( $20 \mathrm{~nm}$ long and of $9 \mathrm{~nm}$ diameter), after 300,000 steps of $0.3 \mathrm{fs}$. The

2-pyridyl-benzimidazole functional group attached to the carbazole has, on the contrary, the possibility

to stack parallel to the surface thanks to the flexibility brought by the alkyl chain.

\section{Influence of the chemical groups attached to the polymer backbone :}

We now address the impact of the functional groups attached to the polymer backbone on the adsorbed pattern and the expected sensing capabilities. For the P1 fluorene:carbazole copolymers made up of 10 and 30 monomers respectively (with 9:1 fluorene:carbazole ratio), and for the P2 polyfluorene polymer (with 9:1 (fluorene carrying probe):fluorene proportion), the physisorption of the polymeric chain on all studied (small to large diameter) CNTs occurs with the same trend as depicted previously, over the same time scale of a few tens of ps. Once the polymer has interacted with the CNT, the two same local configurations for fluorene groups are obtained, with a balanced distribution of parallel $\pi-\pi$ stacked and perpendicular groups. Let us highlight that for the P1 copolymer, the carbazole group is not connected to the neighboring fluorene groups via the usual 2,7 positions in the polymer structure (see figure 1). This introduces angles of about $90^{\circ}$ in the backbone, which is visible for instance in the adsorbed geometries shown in figures 14 or 15 .

Concerning the functional pendant groups, on the one hand, those of P1 are attached to the carbazole units of the backbone and are made up of a unique flexible hexyl chain, ending with a terminal 2-pyridyl-benzimidazole group (with a bicyclic, rigid benzimidazole group attached by a single bond - allowing rotations - to a pyridyl ring). Because of the aromaticity of the carbazole group and the quite low steric hindrance of its functional

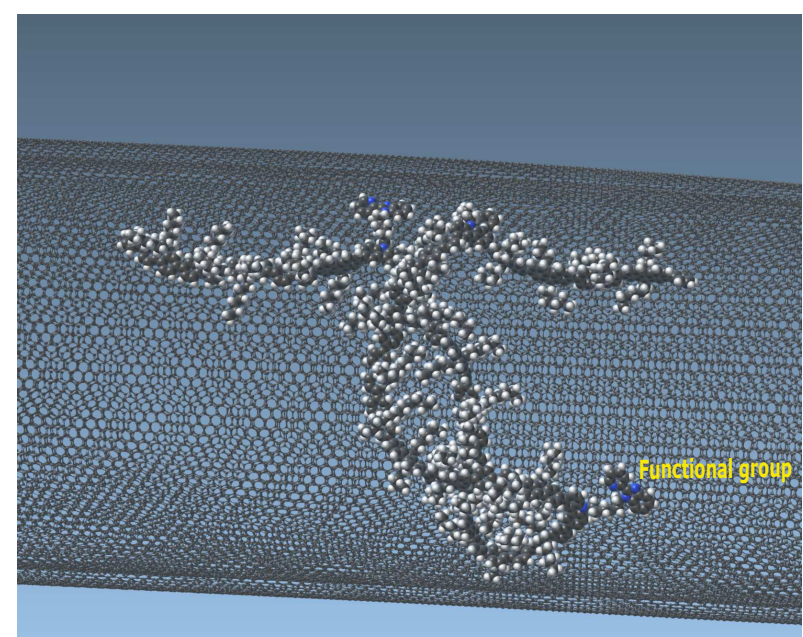

FIG. 12: P1 polymer adsorbed on a Double Walled CNT of $9 \mathrm{~nm}$ outer shell diameter (and $20 \mathrm{~nm}$ long). The inner shell is separated by $3.4 \AA$ from the outer shell.

The polymer is coiled, because of loops on itself prior to the adorption, which end up preventing all monomers from being perfectly adsorbed. The three functional groups are stacked parallel to the CNT sidewalls.

group (one chain only linked to the carbazole group), the pendant group appears to stack parallel to the CNT surface after physisorption - see figures 12, 13, 14, 15, 16 and 20 - except in the case of intra or inter-chain steric repulsion - see figures 16 and 19 respectively. In figure 19, the steric hindrance of one adsorbed polymer hinders the adsorption of almost half of the second polymer chain - on the surface of a $1.7 \mathrm{~nm}$ diameter SWNT. This inter-chain steric hindrance effect seems weaker for larger diameter (e.g. $9 \mathrm{~nm})$ nanotubes, as seen $e . g$. comparing to figure 16 , reporting a possible adsorption configuration of the same two polymers on a $9 \mathrm{~nm}$ diameter nanotube. Although both chains were initially located on the same side (relatively to the CNT), they are both almost wholly adsorbed, due to the much larger surface area and lower surface curvature. The carbazole groups (carrying these P1 functional groups) are mainly stacked to the surface - maximizing their interactions with the underlying CNT surface except when the steric hindrance due to two neighboring perpendicular fluorene groups (attached to two hexyl chains) is too high - see e.g. figure 11 .

On the other hand, the functional groups of $\mathrm{P} 2$ are made of two branches (attached to the same fluorene unit), each one comprising a urea group in-between two phenyl groups. These groups also stack parallel to the CNT (at $3.4 \AA$ from the surface), in the regions where the chain is perfectly extended on the CNT surface, because of $\pi-\pi$ stacking interactions between the phenyl groups and the graphene-like surface (see figure 17). However, in some 


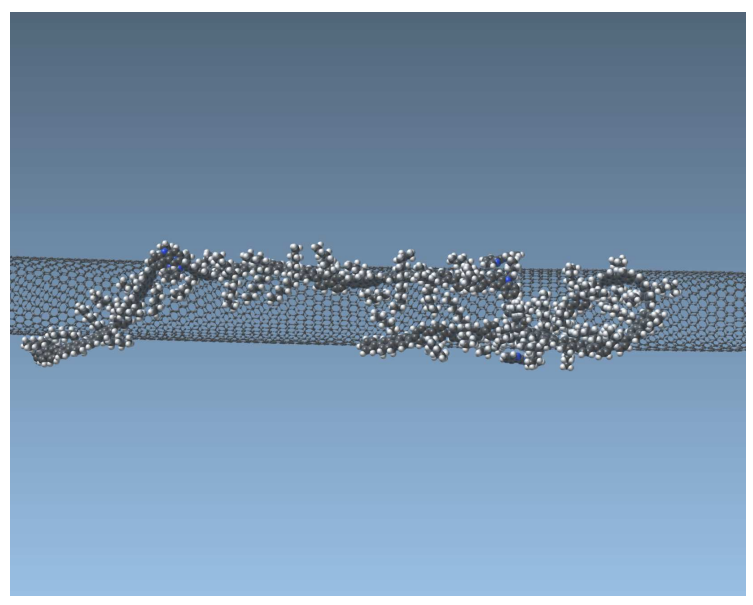

FIG. 13: Possible adsorption geometry of the 30 -monomers long $\mathrm{P} 1$ polymer on a $20 \mathrm{~nm}$ long Single Walled CNT of $1.7 \mathrm{~nm}$ diameter. Similarly to the adsorption example on a Double Wall CNT surface (see figure 12), the polymer makes one loop around itself, preventing all monomers from being adsorbed (region towards the right of the nanotube). Yet, all three functional groups are adsorbed on the surface.

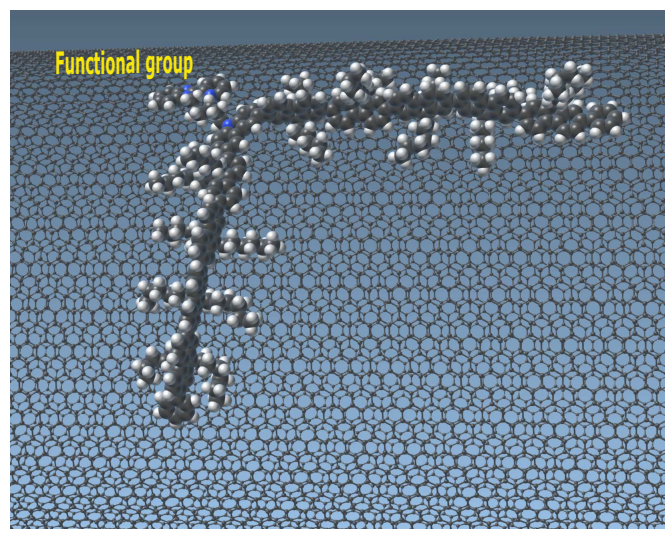

FIG. 14: Configuration of the 10-monomers long $\mathrm{P} 1$ polymer after physisorption on a 20 nm long, 9 nm diameter CNT, after 400,000 steps of $0.3 \mathrm{fs}$ i.e. $120 \mathrm{ps}$. The functional group carried by the chain is adsorbed parallel to the surface. situations - e.g. in the case of coiled adsorbed geometries - these functional groups (belonging to polymer P2) are far from the surface, thus not interacting with it. These local features can be due e.g. to intra-chain interactions - the polymer chain which is underneath prevents some polymer units located further along the backbone from being adsorbed on the sidewalls, see $e$.g. figure 18 .

Similar coiled adsorbed geometries, with intra-chain steric repulsion effects, have been observed as well for P1 polymer adsorbed on a Double Walled CNT (of $9 \mathrm{~nm}$ diameter outer shell) - figure 12 - or on a 1.7 $\mathrm{nm}$ diameter Single Walled CNT - figure 13. In the case of figures 12 and 13, only regular fluorene units were prevented from adsorbing, and not functional groups. Note that these polymers have been designed to recognize some species thanks to the attached functional groups, which will act as probes. The proximity of the latter to the CNT surface appears as a positive point as it is expected to enhance the electronic interaction between the CNT and the complex formed by the probe and the targeted analyte. This should perturbate the electronic properties of the CNT which will act as transducers in the design of ohmic sensors.

Let us also point that no noticeable difference - with respect to the same $(9 \mathrm{~nm})$ outer diameter SWCNT - was observed concerning the average geometrical adsorption pattern of polymers on the Double Walled nanotube surface. The inner nanotube shell interacts only by non-bonded (Coulomb and dispersion vdW) forces with the approaching polymer. These long-range interaction terms however decay fast and are negligible beyond 10 $\AA$. As a consequence, only few atoms of the second shell interact with the atoms of the adsorbed polymer. This may explain that no major structural change is observed. 


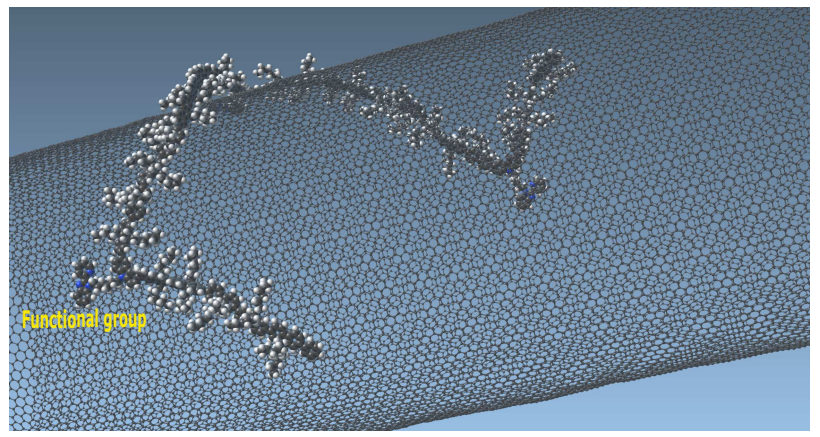

FIG. 15: Adsorption geometry extracted after 300,000 MD steps of $0.3 \mathrm{fs}$, i.e. $90 \mathrm{ps}$ (and further minimized) of the 30-monomers long P1 polymer near a $20 \mathrm{~nm}$ long, 9 $\mathrm{nm}$ diameter CNT. All three functional groups are adsorbed.

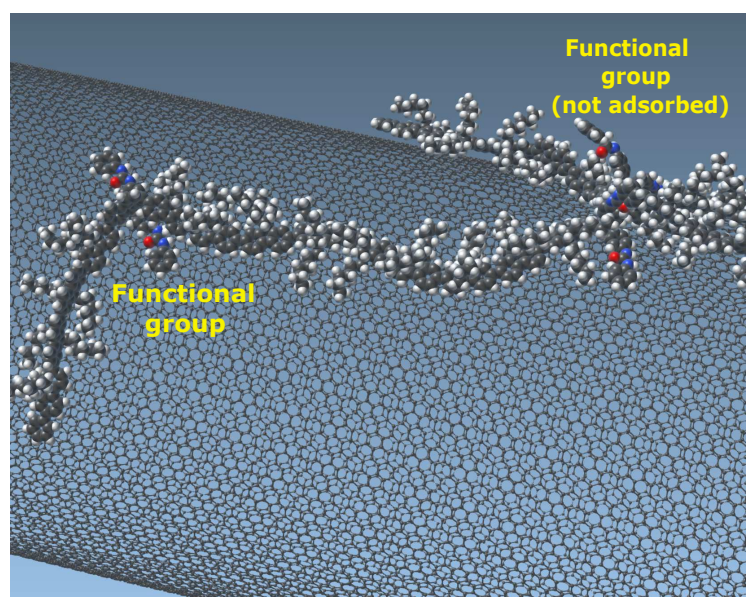

FIG. 17: Example of adsorbed geometry for the 30-monomers long $\mathrm{P} 2$ polymer on a $9 \mathrm{~nm}$ diameter, $20 \mathrm{~nm}$ long CNT. Three functional group branches are adsorbed parallel to the CNT surface, while two

branches are pointing outwards, arguably not interacting with the sidewalls.

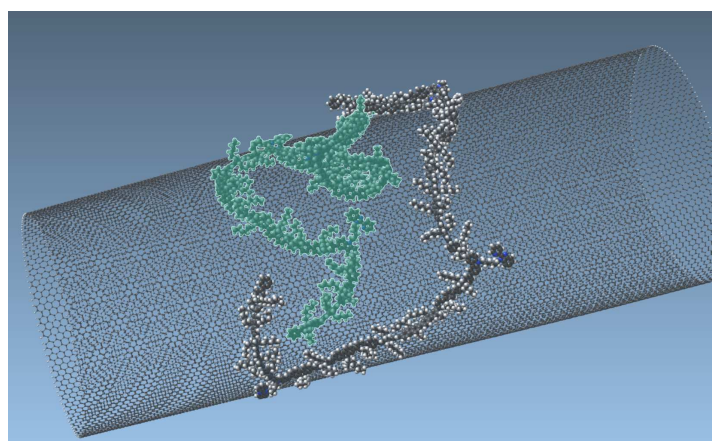

FIG. 16: Adsorption geometry extracted after 400,000 MD steps of 0.3 fs, i.e. 120 ps (and further minimized) of two 30-monomers long P1 polymers, initially on the same side of a $20 \mathrm{~nm}$ long, $9 \mathrm{~nm}$ diameter CNT. While there is almost no inter-chain steric hindrance, intra-chain repulsion occurs for the second, highlighted chain, preventing one of its functional groups from being adsorbed.

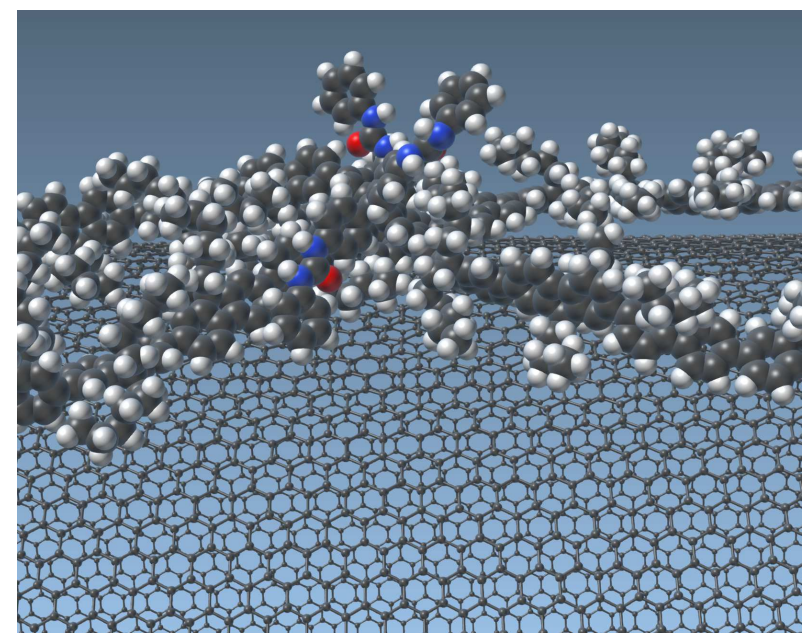

FIG. 18: Example of functional groups (attached to the P2 polymer backbone) not adsorbed on the $9 \mathrm{~nm}$ diameter CNT surface because of intra-chain steric hindrance (due to coiled adsorbed geometries). The sensing of ions by these specific functional groups is likely not to affect the electronic transport properties of the CNT underneath, because of their large distance to the surface.

was described in the previous part for all adsorption geometries. To understand further these observations, we computed the binding energies of the different polymers (but also of some single monomers) on the nanotube surfaces. The results are summarized in Table I.

The adsorption energies of fluorene dihexyl monomers polymer adsorption patterns, and in particular local geometries observed for the fluorene units - main constituents of the polymer backbones. A balanced distribution of perpendicular and parallel fluorene groups 


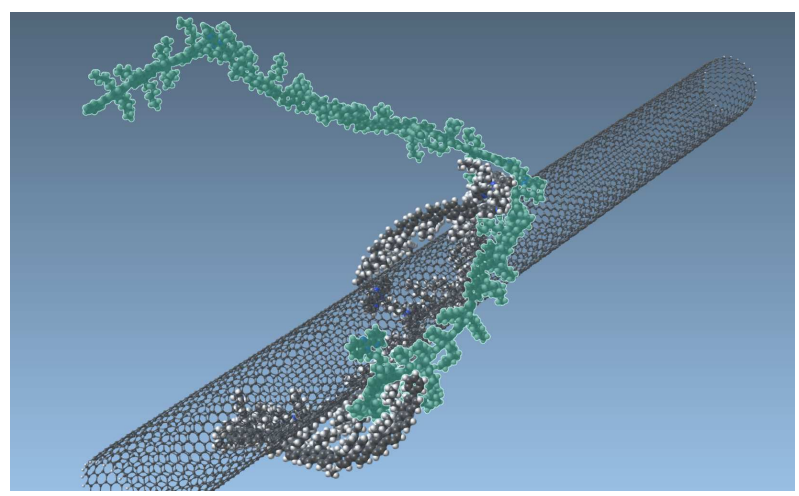

FIG. 19: Example of inter-chain steric hindrance for two 30-monomers long P1 polymers (initially on opposite sides relatively to the nanotube) on a $1.7 \mathrm{~nm}$ diameter, $20 \mathrm{~nm}$ long SWCNT, after 800,000 steps of 0.3 fs i.e. $240 \mathrm{ps}$.

on the CNT surface, when compared to adsorption energies of fluorene dimethyl monomers, allow to isolate the contribution of alkyl chains to the binding energy. Neglecting the binding energies of alkyl chains perpendicular to the CNT surface, we find the following binding energies from the first two lines and the fifth line of Table I :

$$
\begin{gathered}
E_{\text {hexylchain } \| \text { CNTsurface }}^{\text {binding }} \approx-15.5 \mathrm{kcal} / \mathrm{mol} \\
E_{\text {fluoreneunit } \| \text { CNT surface }}^{\text {binding }} \approx-28.1 \mathrm{kcal} / \mathrm{mol} \\
E_{\text {fluorene } \perp \text { CNT surface }}^{\text {binding }} \approx-14.8 \mathrm{kcal} / \mathrm{mol}
\end{gathered}
$$

The value of the binding energy of an hexyl chain parallel to the CNT (i.e. extended along the surface) compares very well to the experimental value of the desorption energy of about $18 \mathrm{kcal} / \mathrm{mol}$ found for an hexyl chain on graphite previously reported. ${ }^{105}$ Chen et al. ${ }^{92}$ reported from numerical simulations using $\mathrm{UFF}^{88}$ a somewhat lower binding energy of about $11 \mathrm{kcal} / \mathrm{mol}$ for octyl chain adsorbed on small SWNTs of $1 \mathrm{~nm}$ diameter - chirality $(7,5)$.

From these results, the parallel fluorene geometry

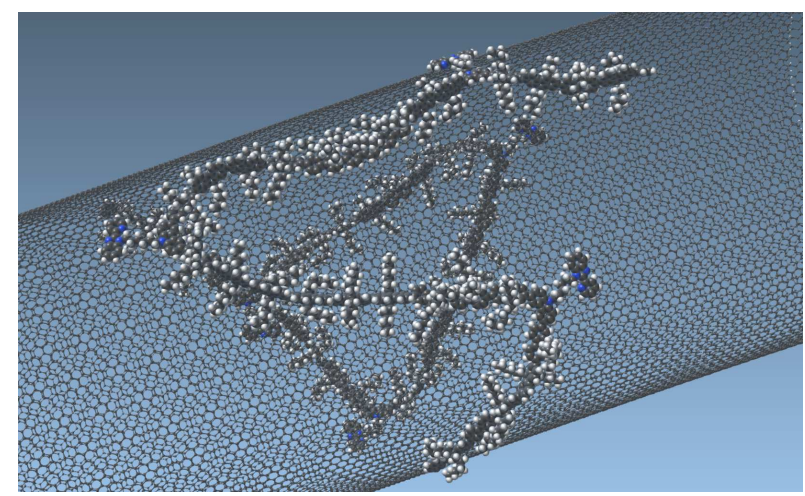

FIG. 20: Example of adsorbed geometry for the two same 30-monomers long P1 polymers (also initially on opposite sides relatively to the nanotube) on a $9 \mathrm{~nm}$ diameter, $20 \mathrm{~nm}$ long SWCNT, after 400,000 steps of 0.3 fs i.e. $120 \mathrm{ps}$. Both chains are fully adsorbed, as well as all functional groups.

appears much more favorable than the perpendicular one. This result explains (at least energetically, at 0 $\mathrm{K}$, overlooking entropic effects) why fluorene units bearing (very short) methyl chains all stack parallel to the nanotube surface - a feature unveiled in the previous section - see figure 10 .

When the fluorene groups bear longer alkyl chains (as for instance hexyl chains), either two hexyl chains are adsorbed along the CNT sidewall when the fluorene unit is in the perpendicular geometry, or only one is if the fluorene is in the parallel geometry. The energetic gain in having one more hexyl chain adsorbed parallel to the surface appears here to exactly compensate the difference in binding energy for the perpendicular $v s$. parallel fluorene configurations (the latter being more favorable than the former without considering hexyl chains). This explains why the binding energies of a single fluorene monomer (bearing two hexyl chains) for the perpendicular and parallel adsorption modes are of the same order of magnitude (see Table I, first two lines), consistently with the results reported for SWNTs. ${ }^{29}$ 


\begin{tabular}{|c|c|c|c|c|c|}
\hline CNT diameter & CNT length & Monomer (or polymer) & Adsorption energy & Main geometric features & Figure \\
\hline $4 \mathrm{~nm}$ & $\begin{array}{l}20 \text { nm (10860 atoms, } \\
\text { hydrogenated) }\end{array}$ & $\begin{array}{l}\text { One perpendicular dihexylfluorene } \\
\text { monomer ( } 59 \text { atoms) }\end{array}$ & $-45.6 \mathrm{kcal} / \mathrm{mol}$ & $\begin{array}{l}\text { Two hexyl chains adsorbed along } \\
\text { the surface, fluorene unit } \\
\text { perpendicular to it. }\end{array}$ & Not shown \\
\hline $4 \mathrm{~nm}$ & $\begin{array}{l}20 \text { nm (10860 atoms, } \\
\text { hydrogenated) }\end{array}$ & $\begin{array}{l}\text { One parallel dihexylfluorene } \\
\text { monomer ( } 59 \text { atoms) }\end{array}$ & $-43.4 \mathrm{kcal} / \mathrm{mol}$ & $\begin{array}{l}\text { One hexyl chain pointing } \\
\text { outwards, one adsorbed along the } \\
\text { surface, fluorene unit parallel to it. }\end{array}$ & Not shown \\
\hline $4 \mathrm{~nm}$ & $20 \mathrm{~nm}$ (10860 atoms) & $\begin{array}{c}15 \text { monomer-long } \\
\text { poly(9,9-dihexylfluorene) } \\
\text { (877 atoms) }\end{array}$ & $\begin{array}{c}-600 \mathrm{kcal} / \mathrm{mol} \\
(-40.0 \mathrm{kcal} / \mathrm{mol} \mathrm{per} \\
\text { monomer })\end{array}$ & $\begin{array}{c}\text { Whole chain adsorbed on the } \\
\text { surface. }\end{array}$ & Figure 4 \\
\hline $4 \mathrm{~nm}$ & $\begin{array}{c}20 \mathrm{~nm} \text { long (10860 } \\
\text { atoms) }\end{array}$ & $\begin{array}{c}30 \text { monomer-long } \\
\text { poly(9,9-dihexylfluorene) } \\
\text { (1732 atoms) }\end{array}$ & $\begin{array}{c}-1135 \mathrm{kcal} / \mathrm{mol} \\
(-37.8 \mathrm{kcal} / \mathrm{mol} \text { per } \\
\text { monomer })\end{array}$ & $\begin{array}{l}\text { Whole chain adsorbed on the } \\
\text { surface. The adsorption pattern is } \\
\text { u-shaped. }\end{array}$ & Figure 8. \\
\hline $4 \mathrm{~nm}$ & $\begin{array}{l}20 \mathrm{~nm} \text { long ( } 10860 \\
\text { atoms, hydrogenated) }\end{array}$ & $\begin{array}{l}\text { One parallel dimethylfluorene } \\
\text { monomer ( } 29 \text { atoms) }\end{array}$ & $-30.8 \mathrm{kcal} / \mathrm{mol}$ & $\begin{array}{c}\text { Dimethylfluorene group parallel to } \\
\text { the surface, one } \mathrm{CH}_{3} \text { unit parallel } \\
\text { to the surface, the other pointing } \\
\text { outwards. }\end{array}$ & Not shown \\
\hline $4 \mathrm{~nm}$ & $\begin{array}{l}20 \mathrm{~nm} \text { long ( } 10860 \\
\text { atoms, hydrogenated) }\end{array}$ & $\begin{array}{c}15 \text { monomer-long } \\
\text { poly(9,9-dimethylfluorene }) \\
\text { (427 atoms) }\end{array}$ & $\begin{array}{l}-416 \mathrm{kcal} / \mathrm{mol} \\
(-27.7 \mathrm{kcal} / \mathrm{mol} \mathrm{per} \\
\text { monomer })\end{array}$ & $\begin{array}{l}\text { S-shaped adsorption geometry, sort } \\
\text { of 'helical wrapping' possibly due } \\
\text { to all fluorene groups being parallel } \\
\text { to the surface. }\end{array}$ & Figure 10. \\
\hline $1.7 \mathrm{~nm}$ & $\begin{array}{l}20 \mathrm{~nm}(5520 \text { atoms, } \\
\text { hydrogenated })\end{array}$ & $\begin{array}{c}30 \text { monomer-long } \mathrm{P} 1 \text { polymer } \\
\text { (1:9 carbazole:fluorene proportion, } \\
1741 \text { atoms })\end{array}$ & $\begin{array}{l}-983 \mathrm{kcal} / \mathrm{mol} \\
(-32.8 \mathrm{kcal} / \mathrm{mol} \text { per } \\
\text { monomer })\end{array}$ & $\begin{array}{l}\text { Intra-polymer steric hindrance: } \\
\text { one 'loop' made by the polymer } \\
\text { before adsorbing (in the MD } \\
\text { exploration), ending up with some } \\
\text { monomers not being in contact } \\
\text { with the surface. }\end{array}$ & Figure 13. \\
\hline $9 \mathrm{~nm}$ & $20 \mathrm{~nm}$ (19473 atoms) & $\begin{array}{l}10 \text { monomer-long P1 polymer } \\
\text { (one functional group, } 9 \text { regular } \\
\text { monomers, } 595 \text { atoms) }\end{array}$ & $\begin{array}{l}-409 \mathrm{kcal} / \mathrm{mol} \\
(-40.9 \mathrm{kcal} / \mathrm{mol} \text { per } \\
\text { monomer })\end{array}$ & $\begin{array}{c}\text { No loop (polymer chain short } \\
\text { enough). Templating effect of the } \\
\text { CNT (the chain adopts the CNT } \\
\text { curvature). }\end{array}$ & Figure 14. \\
\hline $9 \mathrm{~nm}$ & $20 \mathrm{~nm}$ (25870 atoms) & $\begin{array}{l}30 \text { monomer-long P1 polymer } \\
\text { (3 functional groups, } 27 \text { regular } \\
\text { monomers, } 1741 \text { atoms) }\end{array}$ & $\begin{array}{c}-1217 \mathrm{kcal} / \mathrm{mol} \\
(-40.6 \mathrm{kcal} / \mathrm{mol} \text { per } \\
\text { monomer })\end{array}$ & $\begin{array}{c}\text { No loop, chain entirely adsorbed } \\
\text { and adopting the nanotube } \\
\text { curvature. }\end{array}$ & Figure 15 \\
\hline $9 \mathrm{~nm}$ & $\begin{array}{l}20 \text { nm (25870 atoms, } \\
\text { hydrogenated) }\end{array}$ & $\begin{array}{l}\text { Two } 30 \text { monomer-long P1 polymers } \\
\text { (3 'probes', } 27 \text { regular monomers, } \\
1741 \text { atoms each), initially on the } \\
\text { same side of the CNT. }\end{array}$ & $\begin{array}{r}-2347.0 \mathrm{kcal} / \mathrm{mol} \\
(-39.1 \mathrm{kcal} / \mathrm{mol})\end{array}$ & $\begin{array}{l}\text { One loop (intra-chain hindrance) } \\
\text { only for one of the two polymers. }\end{array}$ & Figure 16. \\
\hline $9 \mathrm{~nm}$ & $\begin{array}{l}20 \text { nm (25870 atoms, } \\
\text { hydrogenated) }\end{array}$ & $\begin{array}{c}\text { Two } 30 \text { monomer-long P1 polymers } \\
\text { (3 'probes', } 27 \text { regular monomers, } \\
1741 \text { atoms each), initially on } \\
\text { opposite sides of the CNT. }\end{array}$ & $\begin{array}{r}-2436.0 \mathrm{kcal} / \mathrm{mol} \\
(-40.6 \mathrm{kcal} / \mathrm{mol})\end{array}$ & $\begin{array}{l}\text { Neither intra nor inter chain } \\
\text { hindrance. Both chains fully } \\
\text { adsorbed. }\end{array}$ & Figure 20. \\
\hline $\begin{array}{l}9 \mathrm{~nm} \text { (outer shell), } \\
3.4 \AA \text { distance to } \\
\text { the inner shell } \\
\text { (DWNT) }\end{array}$ & $\begin{array}{c}20 \mathrm{~nm} \text { long (49570 } \\
\text { atoms, both shells } \\
\text { hydrogenated) }\end{array}$ & $\begin{array}{c}30 \text { monomer-long P1 polymer } \\
\text { (3 'probes', } 27 \text { regular monomers, } \\
1741 \text { atoms) }\end{array}$ & $\begin{array}{c}-947 \mathrm{kcal} / \mathrm{mol} \\
(-31.6 \mathrm{kcal} / \mathrm{mol} \text { per } \\
\text { monomer })\end{array}$ & $\begin{array}{l}\text { Very coiled, many loops (the } \\
\text { polymer has time to coil before } \\
\text { reaching the CNT surface } \\
\text { 'template'). }\end{array}$ & Figure 12. \\
\hline $9 \mathrm{~nm}$ & $\begin{array}{c}20 \mathrm{~nm}(25870 \text { atoms, } \\
\text { hydrogenated })\end{array}$ & $\begin{array}{c}30 \text { monomer-long P2 polymer } \\
\text { (3 'probes', } 27 \text { regular monomers, } \\
1780 \text { atoms), 'perpendicular' initial } \\
\text { geometry }\end{array}$ & $\begin{array}{l}-937 \mathrm{kcal} / \mathrm{mol} \\
(-31.0 \mathrm{kcal} / \mathrm{mol} \text { per } \\
\text { monomer })\end{array}$ & $\begin{array}{l}\text { Very coiled, many loops (the } \\
\text { polymer, being 'perpendicular' to } \\
\text { the CNT axis initially, has time to } \\
\text { coil before reaching the CNT } \\
\text { surface 'template'). }\end{array}$ & Figures $17,18$. \\
\hline
\end{tabular}

TABLE I: Estimated adsorption energies derived from structural minimizations (with ReaxFF) for fluorene monomers - perpendicular and parallel to the CNT surface - and for whole polymer chains adsorbed on CNTs. The geometries are all extracted from NVT molecular dynamics simulations, once the interaction is complete, and further minimized (at $0 \mathrm{~K}$ ). Binding energies are then derived by substracting the energies of both

the isolated polymer and CNT (each minimized alone at $0 \mathrm{~K}$ ). Per monomer adsorption energies are obtained by dividing the total adsorption energy of the polymer chain by its number of monomers, neglecting the contribution of the two terminal phenyl groups at both chain ends. 
We observed the following interesting features on the calculated adsorption energies. For coiled adsorbed geometries, the average binding energy per monomer is of order $32 \mathrm{kcal} / \mathrm{mol}$ for both polymers (with no noticeable difference for the adsorption on the Double Walled nanotube). This is significantly lower than for 'ideally' adsorbed polymer geometries, with the whole chain being in contact with the CNT sidewalls, with an average binding energy of $40 \mathrm{kcal} / \mathrm{mol}$ per monomer. This adsorption energy per monomer unit is closer to the maximum binding energy per fluorene monomer (bearing two hexyl chains), found to be of order 45 $\mathrm{kcal} / \mathrm{mol}$ in the ideal case of a single monomer unit adsorbed on the CNT surface (two first lines of Table I). The presence of some units in the polymer backbone bearing functional groups can also be accountable for the variability of the adsorption energies (compared to the ideal case).

The binding energies reported in Table I for different polymers or monomers are typical of non-covalent functionalization. Indeed, the binding energies per monomer range from 1.3 to $1.7 \mathrm{eV}$, which in turn converts to about 20 to $30 \mathrm{meV} /$ atom (for monomers of 59 atoms), a typical energy for weak $\pi-\pi$ stacking interaction. It is consistent with values of about $1 \mathrm{eV}$ reported in Ref. $^{47}$ (obtained at the DFTB level ${ }^{106}$ ) for polyfluorenes bearing dodecyl side chains adsorbed on SWNT of about $1 \mathrm{~nm}$ diameter and $50 \AA$ long. It is also consistent with the binding energies of $0.5 \mathrm{eV}$ per ADN nucleobase (small units containing one or two aromatic rings, of size comparable to fluorene monomers) derived in Ref. ${ }^{45}$ in solvated conditions (using AMBER force field), and with the total adsorption energies - of $300 \mathrm{kcal} / \mathrm{mol}$, i.e. 13 $\mathrm{eV}$, in total - derived for shorter stiff-backbone polymers on SWNTs ${ }^{91}$ (using DREIDING force field).

Binding energies per unit length of nanotubes have also been reported to range from 15 to $20 \mathrm{kcal} /(\mathrm{mol} . \AA$ ) in Ref. $^{29}$ (in the case of multiple polyfluorene chains, bearing alkyl chains of varying length, adsorbed on SWNTs of about $1 \mathrm{~nm}$ diameter, and covering almost the whole surface) using CVFF force field, and from 6 to 12 $\mathrm{kcal} /(\mathrm{mol} . \AA$ ) $)$ for one poly(9,9-dioctylfluorene) adsorbed on $\mathrm{SWNT}^{25}$ (using MM3 force field).

In our simulations with $20 \mathrm{~nm}$ long, small $(1.7 \mathrm{~nm})$ to large $(9 \mathrm{~nm})$ diameter CNTs, the adsorption energy for a single polymer made up of 30 units is of order 1000 $\mathrm{kcal} / \mathrm{mol}$, and 2000 to $2400 \mathrm{kcal} / \mathrm{mol}$ for two polymers (see Table I). Thus the binding energy per unit length of CNT found here with ReaxFF is in the order of 5 $\mathrm{kcal} /(\mathrm{mol} . \AA)$ for one adsorbed polymer and twice as much for two adsorbed polymers. Binding energies on a given nanotube are mainly additive, e.g. when increasing the length of a polymer chain (or as well when increasing the length of the alkyl chains), as they are driven by van der Waals dispersion forces (a feature proved in the SI for smaller model systems), which are additive. However, because of steric repulsion between polymers (see e.g. figure 19), and entropic reasons, this binding energy by unit length might not increase perfectly linearly with the number of neighboring polymers.

\section{Focus on guided polymer selection :}

These ReaxFF-based MD simulations show that the investigated polymers are able to functionalize CNTs, although in many different ways. We have studied the influence on the CNT/polymer geometry of the alkyl chain length and functional groups carried by the polymer backbone. We have also highlighted the existence of coiled adsorption geometries for long polymer chains due to intra-chain, or inter-chain (in the case of multiple polymers) steric hindrance. The differences in adsorption geometries and energies observed for different polymer types (and lengths) in this study can lead to an 'optimal' polymer selection (among a given set of candidates), with respect to a given purpose. Let us illustrate the polymer selection methodology enabled by these simulations. We aim at enhancing the sensitivity of the CNT electronic properties towards a target molecule or a target ion (in solvent) captured by the probe of the polymer $-e . g$. as targeted in Ref. ${ }^{31,32}$ Thus, we are looking for a maximal adsorption of the polymer on the CNT surface, without intrachain coiling, so that the functional groups are close enough to the surface. In that case, the complexation of a target ion by a functional group adsorbed on the surface can modulate the conduction properties of the nanotube. The functional groups have also, if possible, to interact only weakly with the CNT surface - to preserve their ion binding capabilities. These objectives imply, according to the previous results, to design a rather short polymer (oligomer) to lower the probability of coiled adsorbed geometries. Indeed, we have seen that the proximity of the sensing probes to the CNT surface is favored by a locally complete adsorption pattern of the polymer chain, avoiding any loop or intrachain coiling, which is more likely for shorter polymer chains (for entropic reasons of intrachain coiling possibilities).

\section{CONCLUSIONS}

Thanks to ReaxFF-based MD simulations, we estimated the main geometrical features of non-covalent functionalization of small $(1.7 \mathrm{~nm})$ to large $(9 \mathrm{~nm}) \mathrm{di}$ ameter Single and Double Walled CNTs - representative of most classes of possible nanotubes outer shells - by polyfluorene homopolymers and carbazole:fluorene copolymers, carrying functional groups targeted at specific ions. Two main local adsorption geometries were unveiled for fluorene units (namely perpendicular and parallel), generalizing similar results derived in previous 
articles for SWNTs, of about $1 \mathrm{~nm}$ diameter, only. ${ }^{29,47}$ Moreover, an energetic explanation is provided to these features, and the role of the length of the alkyl chains grafted to the polyfluorene backbone was clarified. Alkyl chains which are short enough, such as methyl groups, lead to adsorption of all the units of the chain in the parallel mode only. The possible adsorption equilibrium geometries for the functional groups periodically grafted to the polymer backbone have also been analyzed on two polymers of similar structure, but bearing functional groups of different nature (and different steric hindrance). Both functional groups studied tend to adsorb parallel to the CNT surface (thanks to $\pi-\pi$ interactions) due to their aromatic character, when possible, i.e. outside of any local intrachain (loop) or interchain steric hindrance preventing complete adsorption.

We have also analyzed coiled adsorption geometries for (long enough) polymers on CNT surfaces, while most previous studies only reported and described the features of the ideal case of whole chain adsorption, or of helical wrapping (on SWNTs). These observed coilings of the polymer over the CNT sidewalls were explained through the particular choices of initial geometries of the polymer relative to the CNT. When the former is far enough from the CNT, the MD exploration is likely to lead to loops and intra-chain interactions (because of purely entropic reasons) prior to the adsorption and the irreversible 'templating' effect of the CNT. These complex and coiled adsorption geometries are the ones most likely happening in real conditions, not withstanding the presence of other polymers, adding interchain interactions prior to and during the adsorption (which can result far from a perfect pattern of complete CNT/polymer interaction, as shown above).

To validate the use of ReaxFF force field (and of the specific parametrization used here ${ }^{48}$ ), we performed benchmark calculations on model systems and compared the geometries and energies derived to the results on similar systems within higher levels of theory (Supporting Information). We showed that the correct binding geometries and correct order of magnitude for binding energies in $\pi-\pi$ stacking systems are derived. However, we highlighted that the relative orientation of the polymer chain with respect to the CNT surface lattice can not be discussed here with ReaxFF, as it does not capture the translational energy barrier upon displacement of polycyclic aromatic molecules parallel to the flat surface.

The methodology associated to this study, to derive adsorption geometries and energies of various polymer or oligomer chains (without any re-parametrization effort, i.e. no specific new parameters are needed to simulate our new systems) can be a valuable tool for optimal polymer design. Some examples of possible side chain (alkyl chains, or functional groups) fine-tuning were given, in the perspective of current CNT/polymer compounds typical applications.
This work also opens several perspectives. Our simulation methodology paves the way towards further understanding of steric effects of polymers adsorption on different diameter nanotubes. The average nanotube surface coverage (by neighboring polymers), as a function of nanotube diameter, number of polymers, length of polymer chains, and (possibly) length of alkyl chains, is directly depending on the competition between intrachain, inter-chain and polymer-nanotube interactions. It might be an enlightening quantity, of experimental significance, to derive from further simulations. Finally, ab-initio techniques launched on fixed geometries, previously extracted from molecular dynamics simulations and further relaxed, could help shed light on the possible subtle charge redistribution happening upon non-covalent functionalization of a CNT by a conjugated polymer. The precise origin of a possible 'doping' (or 'charge transfer') by molecular physisorption mentioned in numerous articles, ${ }^{107}$ and the level of description needed to capture these effects correctly (rather in terms of electronic density, polarizable force field, or simple fixed partial charges model) remains to be discussed.

\section{SUPPLEMENTARY MATERIAL}

See Supplementary Material (Supporting Information) for the validation of ReaxFF force field and of its specific parametrization, thanks to benchmark calculations on model systems and comparison to quantum calculations.

${ }^{1}$ Fulvio Michelis, Laurence Bodelot, Yvan Bonnassieux, and Bérengère Lebental. Highly reproducible, hysteresis-free, flexible strain sensors by inkjet printing of carbon nanotubes. Carbon, 95:1020-1026, dec 2015.

${ }^{2}$ G. U. Sumanasekera, B. K. Pradhan, H. E. Romero, K. W. Adu, and P. C. Eklund. Giant thermopower effects from molecular physisorption on carbon nanotubes. Physical Review Letters, 89(16), sep 2002 .

${ }^{3}$ Joseph Wang. Carbon-nanotube based electrochemical biosensors: A review. Electroanalysis, 17(1):7-14, jan 2005.

${ }^{4}$ Robert A. Bell, Michael C. Payne, and Arash A. Mostofi. Does water dope carbon nanotubes? The Journal of Chemical Physics, 141(16): 164703, oct 2014.

${ }^{5}$ Agnieszka Lekawa-Raus, Lukasz Kurzepa, Gregory Kozlowski, Simon C. Hopkins, Mariusz Wozniak, Damian Lukawski, Bartek A. Glowacki, and Krzysztof K. Koziol. Influence of atmospheric water vapour on electrical performance of carbon nanotube fibres. Carbon, 87:18-28, jun 2015.

${ }^{6} \mathrm{~J}$. Kong. Nanotube molecular wires as chemical sensors. Science, 287(5453):622-625, jan 2000.

${ }^{7}$ E. S. Snow. Chemical detection with a single-walled carbon nanotube capacitor. Science, 307(5717):1942-1945, mar 2005.

${ }^{8}$ Joshua A. Robinson, Eric S. Snow, Stefan C. Badescu, Thomas L. Reinecke, and F. Keith Perkins. Role of defects in single-walled carbon nanotube chemical sensors. Nano Letters, 6(8):1747-1751, aug 2006.

${ }^{9}$ Vera Schroeder, Suchol Savagatrup, Maggie He, Sibo Lin, and Timothy M. Swager. Carbon nanotube chemical sensors. Chemical Reviews, 119(1):599-663, sep 2018. 
${ }^{10}$ Yan Li, Miroslav Hodak, Wenchang Lu, and J. Bernholc. Mechanisms of NH3 and NO2 detection in carbon-nanotube-based sensors: An ab initio investigation. Carbon, 101:177-183, may 2016.

${ }^{11}$ A. R. Rocha, M. Rossi, A. Fazzio, and Antonio J. R. da Silva. Designing real nanotube-based gas sensors. Physical Review Letters, 100(17), apr 2008.

${ }^{12}$ Kyongsoo Lee, Jae-Hong Kwon, Seung 11 Moon, Woo-Sung Cho, Byeong-Kwon Ju, and Yun-Hi Lee. pH sensitive multiwalled carbon nanotubes. Materials Letters, 61(14-15):3201-3203, jun 2007.

${ }^{13}$ Wei Zhao, Chulho Song, and Pehr E. Pehrsson. Water-soluble and optically $\mathrm{pH}$-sensitive single-walled carbon nanotubes from surface modification. Journal of the American Chemical Society, 124(42):12418-12419, oct 2002.

${ }^{14}$ Tsuyohiko Fujigaya and Naotoshi Nakashima. Non-covalent polymer wrapping of carbon nanotubes and the role of wrapped polymers as functional dispersants. Science and Technology of Advanced Materials, 16(2):024802, apr 2015.

${ }^{15}$ Christopher A. Hunter and Jeremy K. M. Sanders. The nature of $\pi-\pi$ interactions. Journal of the American Chemical Society, 112(14):5525-5534, jul 1990.

${ }^{16}$ Iwona Pelech, Robert Pelech, Agnieszka Kaczmarek, Anna Jedrzejewska, and Dariusz Moszynski. Effect of treating method on the physicochemical properties of amine-functionalized carbon nanotubes. International Journal of Materials Research, 107(1):35-43, jan 2016.

${ }^{17}$ R. M. Tromp, A. Afzali, M. Freitag, D. B. Mitzi, and Zh. Chen. Novel strategy for diameter-selective separation and functionalization of single-wall carbon nanotubes. Nano Letters, 8(2):469-472, feb 2008.

${ }^{18}$ Jeong-Yuan Hwang, Adrian Nish, James Doig, Sigrid Douven, Chun-Wei Chen, Li-Chyong Chen, and Robin J. Nicholas. Polymer structure and solvent effects on the selective dispersion of singlewalled carbon nanotubes. Journal of the American Chemical Society, 130(11):3543-3553, mar 2008.

${ }^{19}$ Chandrani Pramanik, Jacob R. Gissinger, Satish Kumar, and Hendrik Heinz. Carbon nanotube dispersion in solvents and polymer solutions: Mechanisms, assembly, and preferences. ACS Nano, 11(12):12805-12816, nov 2017.

${ }^{20}$ Fuming Chen, Bo Wang, Yuan Chen, and Lain-Jong Li. Toward the extraction of single species of single-walled carbon nanotubes using fluorene-based polymers. Nano Letters, 7(10):3013-3017, oct 2007.

${ }^{21}$ Fabien A. Lemasson, Timo Strunk, Peter Gerstel, Frank Hennrich, Sergei Lebedkin, Christopher Barner-Kowollik, Wolfgang Wenzel, Manfred M. Kappes, and Marcel Mayor. Selective dispersion of single-walled carbon nanotubes with specific chiral indices by poly(n-decyl-2,7-carbazole). Journal of the American Chemical Society, 133(4):652-655, feb 2011.

${ }^{22}$ Gerald J. Brady, Yongho Joo, Meng-Yin Wu, Matthew J. Shea, Padma Gopalan, and Michael S. Arnold. Polyfluorene-sorted, carbon nanotube array field-effect transistors with increased current density and high on/off ratio. ACS Nano, 8(11):11614-11621, nov 2014.

${ }^{23}$ Widianta Gomulya, Jia Gao, and Maria Antonietta Loi. Conjugated polymer-wrapped carbon nanotubes: physical properties and device applications. The European Physical Journal B, 86(10), oct 2013.

${ }^{24}$ Po-I Wang, Chou-Yi Tsai, Yung-Jou Hsiao, Jyh-Chiang Jiang, and Der-Jang Liaw. High-purity semiconducting single-walled carbon nanotubes via selective dispersion in solution using fully conjugated polytriarylamines. Macromolecules, 49(22):8520-8529, nov 2016.

${ }^{25}$ Adrian Nish, Jeong-Yuan Hwang, James Doig, and Robin J. Nicholas. Highly selective dispersion of single-walled carbon nanotubes using aromatic polymers. Nature Nanotechnology, 2(10):640-646, sep 2007.

${ }^{26}$ Florian Boon, Simon Desbief, Lorenzo Cutaia, Olivier Douhéret, Andrea Minoia, Benoît Ruelle, Sébastien Clément, Olivier Coulembier, Jérôme Cornil, Philippe Dubois, and Roberto Lazzaroni. Synthesis and characterization of nanocomposites based on functional regioregular poly(3-hexylthiophene) and multiwall carbon nanotubes. Macromolecular Rapid Communications, 31(16):14271434, aug 2010.

${ }^{27}$ Wei Zhi Wang, Wei Feng Li, Xiao Yong Pan, Chang Ming Li, LainJong Li, Yu Guang Mu, John A Rogers, and Mary B. Chan-Park. Degradable conjugated polymers: Synthesis and applications in enrichment of semiconducting single-walled carbon nanotubes. Advanced Functional Materials, 21(9):1643-1651, mar 2011.

${ }^{28}$ Jia Gao, Maria Antonietta Loi, Elton José Figueiredo de Carvalho, and Maria Cristina dos Santos. Selective wrapping and supramolecular structures of polyfluorene-carbon nanotube hybrids. ACS Nano, 5(5):3993-3999, may 2011.

${ }^{29}$ Widianta Gomulya, Guadalupe Diaz Costanzo, Elton J. Figueiredo de Carvalho, Satria Zulkarnaen Bisri, Vladimir Derenskyi, Martin Fritsch, Nils Fröhlich, Sybille Allard, Pavlo Gordiichuk, Andreas Herrmann, Siewert Jan Marrink, Maria Cristina dos Santos, Ulrich Scherf, and Maria Antonietta Loi. Semiconducting single-walled carbon nanotubes on demand by polymer wrapping. Advanced Materials, 25(21):2948-2956, apr 2013.

${ }^{30}$ Shinsuke Ishihara, Joseph M. Azzarelli, Markrete Krikorian, and Timothy M. Swager. Ultratrace detection of toxic chemicals: Triggered disassembly of supramolecular nanotube wrappers. Journal of the American Chemical Society, 138(26):8221-8227, jun 2016.

${ }^{31}$ Gaël Zucchi, Bérengère Lebental, Loïc Loisel, Sasikumar Ramachandran, Alfredo Flores Guttierez, Xin Yang Wang, Mallesham Godumala, and Laurence Bodelot. Fr 1753131 - capteurs chimiques à base de nanotubes de carbone fonctionnalisés par des polymères conjugués pour l'analyse en milieu aqueux, January 2017.

${ }^{32}$ Bérengère Lebental, Robert Benda, Laurence Bodelot, Ileana Florea, Mallesham Godumala, Boris Gusarov, Alfredo Flores Guttierez, Loïc Loisel, Erick Merliot, Sasikumar Ramachandran, Xin Yang Wang, and Gaël Zucchi. Carbon nanotube sensor array for water monitoring with conjugated polymers. In C'NANO 2017, The Nanoscience Meeting, Dec. 2017, Lyon, France, 2017.

${ }^{33}$ X.Q. He, S. Kitipornchai, and K.M. Liew. Buckling analysis of multi-walled carbon nanotubes: a continuum model accounting for van der waals interaction. Journal of the Mechanics and Physics of Solids, 53(2):303-326, feb 2005.

${ }^{34} \mathrm{Kun} \mathrm{Li}$ and Boqin Gu. Molecular dynamics investigation of the physisorption and interfacial characteristics of NBR chains on carbon nanotubes with different characteristics. AIP Advances, 7(7):075106, jul 2017.

${ }^{35}$ Yupeng Li, Hyung ick Kim, Bingqing Wei, Junmo Kang, Jae boong Choi, Jae-Do Nam, and Jonghwan Suhr. Understanding the nanoscale local buckling behavior of vertically aligned MWCNT arrays with van der waals interactions. Nanoscale, 7(34):1429914304, 2015.

${ }^{36}$ Samuel D. Stranks, Severin N. Habisreutinger, Beate Dirks, and Robin J. Nicholas. Novel carbon nanotube-conjugated polymer nanohybrids produced by multiple polymer processing. Advanced Materials, 25(31):4365-4371, may 2013.

${ }^{37}$ Marco Bernardi, Michele Giulianini, and Jeffrey C. Grossman. Selfassembly and its impact on interfacial charge transfer in carbon nanotube/p3ht solar cells. ACS Nano, 4(11):6599-6606, oct 2010.

${ }^{38}$ Céline Bounioux, Eugene A. Katz, and Rachel Yerushalmi Rozen. Conjugated polymers - carbon nanotubes-based functional materials for organic photovoltaics: a critical review. Polymers for Advanced Technologies, 23(8):1129-1140, jul 2012.

${ }^{39}$ Yosuke Kanai and Jeffrey C. Grossman. Role of semiconducting and metallic tubes in p3ht/carbon-nanotube photovoltaic heterojunctions: Density functional theory calculations. Nano Letters, 8(3):908-912, mar 2008.

${ }^{40}$ Florian Massuyeau, Jany Wéry, Jean-Luc Duvail, Serge Lefrant, Abu Yaya, Chris Ewels, and Eric Faulques. Electronic interaction in composites of a conjugated polymer and carbon nanotubes: firstprinciples calculation and photophysical approaches. Beilstein Journal of Nanotechnology, 6:1138-1144, may 2015.

${ }^{41}$ John Fennell, Hitoshi Hamaguchi, Bora Yoon, and Timothy Swager. Chemiresistor devices for chemical warfare agent detection 
based on polymer wrapped single-walled carbon nanotubes. Sensors, 17(5):982, apr 2017.

${ }^{42}$ Mazzura Wan Chik, Zahid Hussain, Mohd Zulkefeli, Minaketan Tripathy, Sunil Kumar, Abu Bakar Abdul Majeed, and K. Byrappa. Polymer-wrapped single-walled carbon nanotubes: a transformation toward better applications in healthcare. Drug Delivery and Translational Research, 9(2):578-594, mar 2018.

${ }^{43}$ Fei Wang, Hongwei Gu, and Timothy M. Swager. Carbon nanotube/polythiophene chemiresistive sensors for chemical warfare agents. Journal of the American Chemical Society, 130(16):53925393, apr 2008.

${ }^{44}$ Fei Wang, Yong Yang, and Timothy M. Swager. Molecular recognition for high selectivity in carbon nanotube/polythiophene chemiresistors. Angewandte Chemie International Edition, 47(44):83948396, oct 2008.

${ }^{45}$ Robert R. Johnson, A. T. Charlie Johnson, and Michael L. Klein. Probing the structure of DNA-carbon nanotube hybrids with molecular dynamics. Nano Letters, 8(1):69-75, jan 2008.

${ }^{46}$ Lenibel Santiago-Rodríguez, Germarie Sánchez-Pomales, and Carlos R. Cabrera. DNA-functionalized carbon nanotubes: Synthesis, self-assembly, and applications. Israel Journal of Chemistry, 50(3):277-290, sep 2010.

${ }^{47}$ Jorge Mario Salazar-Rios, Wytse Talsma, Vladimir Derenskyi, Widianta Gomulya, Tina Keller, Martin Fritsch, Sebastian Kowalski, Eduard Preis, Ming Wang, Sybille Allard, Guillermo Carlos Bazan, Ullrich Scherf, Maria Cristina dos Santos, and Maria Antonietta Loi. Understanding the selection mechanism of the polymer wrapping technique toward semiconducting carbon nanotubes. Small Methods, 2(4):1700335, feb 2018.

${ }^{48}$ Alejandro Strachan, Adri C. T. van Duin, Debashis Chakraborty, Siddharth Dasgupta, and William A. Goddard. Shock waves in highenergy materials: The initial chemical events in nitramine RDX. Physical Review Letters, 91(9), aug 2003.

${ }^{49} \mathrm{~F}$. Tournus and J.-C. Charlier. Ab initiostudy of benzene adsorption on carbon nanotubes. Physical Review B, 71(16), apr 2005.

${ }^{50} \mathrm{~F}$. Tournus, S. Latil, M. I. Heggie, and J.-C. Charlier. $\pi$-stacking interaction between carbon nanotubes and organic molecules. Physical Review B, 72(7), aug 2005.

${ }^{51}$ Sergey M. Kozlov, Francesc Viñes, and Andreas Görling. On the interaction of polycyclic aromatic compounds with graphene. Carbon, 50(7):2482-2492, jun 2012.

${ }^{52}$ Henna Ruuska and Tapani A. Pakkanen. Ab initio study of interlayer interaction of graphite: benzene-coronene and coronene dimer twolayer models. The Journal of Physical Chemistry B, 105(39):95419547, oct 2001

${ }^{53}$ F. Ortmann, F. Bechstedt, and W. G. Schmidt. Semiempirical van der waals correction to the density functional description of solids and molecular structures. Physical Review B, 73(20), may 2006.

${ }^{54}$ Renju Zacharia, Hendrik Ulbricht, and Tobias Hertel. Interlayer cohesive energy of graphite from thermal desorption of polyaromatic hydrocarbons. Physical Review B, 69(15), apr 2004.

${ }^{55}$ Olga V. Ershova, Timothy C. Lillestolen, and Elena Bichoutskaia. Study of polycyclic aromatic hydrocarbons adsorbed on graphene using density functional theory with empirical dispersion correction. Physical Chemistry Chemical Physics, 12(24):6483, 2010.

${ }^{56}$ Jeng-Da Chai and Martin Head-Gordon. Long-range corrected hybrid density functionals with damped atom-atom dispersion corrections. Physical Chemistry Chemical Physics, 10(44):6615, 2008.

${ }^{57}$ Stefan Grimme. Semiempirical GGA-type density functional constructed with a long-range dispersion correction. Journal of Сотриtational Chemistry, 27(15):1787-1799, 2006.

${ }^{58}$ Petr Jurečka, Jiř́ Š Šponer, Jiřŕ Černý, and Pavel Hobza. Benchmark database of accurate (MP2 and CCSD(T) complete basis set limit) interaction energies of small model complexes, DNA base pairs, and amino acid pairs. Phys. Chem. Chem. Phys., 8(17):1985-1993, 2006.

${ }^{59}$ Stefan Grimme. Density functional theory with london dispersion corrections. Wiley Interdisciplinary Reviews: Computational Molecular Science, 1(2):211-228, mar 2011.
${ }^{60}$ Jonas Björk, Felix Hanke, Carlos-Andres Palma, Paolo Samori, Marco Cecchini, and Mats Persson. Adsorption of aromatic and antiaromatic systems on graphene through $\pi-\pi$ stacking. The Journal of Physical Chemistry Letters, 1(23):3407-3412, nov 2010.

${ }^{61}$ Steven Bailey, David Visontai, Colin J. Lambert, Martin R. Bryce, Harry Frampton, and David Chappell. A study of planar anchor groups for graphene-based single-molecule electronics. The Journal of Chemical Physics, 140(5):054708, feb 2014.

${ }^{62}$ Yufeng Guo and Wanlin Guo. Interlayer energy-optimum stacking registry for two curved graphene sheets of nanometre dimensions. Molecular Simulation, 34(8):813-819, jul 2008.

${ }^{63}$ Yasushi Shibuta and James A. Elliott. Interaction between two graphene sheets with a turbostratic orientational relationship. Chemical Physics Letters, 512(4-6):146-150, aug 2011.

${ }^{64}$ J.-C Charlier, X Gonze, and J.-P Michenaud. Graphite interplanar bonding: Electronic delocalization and van der waals interaction. Europhysics Letters (EPL), 28(6):403-408, nov 1994.

${ }^{65} \mathrm{E}$. Mostaani, N.D. Drummond, and V.I. Fal'ko. Quantum monte carlo calculation of the binding energy of bilayer graphene. Physical Review Letters, 115(11), sep 2015.

${ }^{66}$ Mark A. Vincent and Ian H. Hillier. Accurate prediction of adsorption energies on graphene, using a dispersion-corrected semiempirical method including solvation. Journal of Chemical Information and Modeling, 54(8):2255-2260, aug 2014.

${ }^{67}$ Irina V. Lebedeva, Andrey A. Knizhnik, Andrey M. Popov, Yurii E. Lozovik, and Boris V. Potapkin. Interlayer interaction and relative vibrations of bilayer graphene. Physical Chemistry Chemical Physics, 13(13):5687, 2011.

${ }^{68}$ Weizhou Wang, Tao Sun, Yu Zhang, and Yi-Bo Wang. Substituent effects in the $\pi-\pi$ interaction between graphene and benzene: An indication for the noncovalent functionalization of graphene. Computational and Theoretical Chemistry, 1046:64-69, oct 2014.

${ }^{69}$ Vincenzo Barone, Maurizio Casarin, Daniel Forrer, Michele Pavone, Mauro Sambi, and Andrea Vittadini. Role and effective treatment of dispersive forces in materials: Polyethylene and graphite crystals as test cases. Journal of Computational Chemistry, 30(6):934-939, apr 2009.

${ }^{70}$ M. Dion, H. Rydberg, E. Schröder, D. C. Langreth, and B. I. Lundqvist. Van der waals density functional for general geometries. Physical Review Letters, 92(24), jun 2004.

${ }^{71}$ Yong-Hui Zhang, Kai-Ge Zhou, Ke-Feng Xie, Jing Zeng, Hao-Li Zhang, and Yong Peng. Tuning the electronic structure and transport properties of graphene by noncovalent functionalization: effects of organic donor, acceptor and metal atoms. Nanotechnology, 21(6):065201, jan 2010.

${ }^{72}$ Evgeniy G. Gordeev, Mikhail V. Polynski, and Valentine P. Ananikov. Fast and accurate computational modeling of adsorption on graphene: a dispersion interaction challenge. Physical Chemistry Chemical Physics, 15(43):18815, 2013.

${ }^{73}$ Weizhou Wang, Yu Zhang, and Yi-Bo Wang. Noncovalent $\pi-\pi$ interaction between graphene and aromatic molecule: Structure, energy, and nature. The Journal of Chemical Physics, 140(9):094302, mar 2014.

${ }^{74}$ A. D. MacKerell, D. Bashford, M. Bellott, R. L. Dunbrack, J. D. Evanseck, M. J. Field, S. Fischer, J. Gao, H. Guo, S. Ha, D. JosephMcCarthy, L. Kuchnir, K. Kuczera, F. T. K. Lau, C. Mattos, S. Michnick, T. Ngo, D. T. Nguyen, B. Prodhom, W. E. Reiher, B. Roux, M. Schlenkrich, J. C. Smith, R. Stote, J. Straub, M. Watanabe, J. Wiórkiewicz-Kuczera, D. Yin, and M. Karplus. All-atom empirical potential for molecular modeling and dynamics studies of proteins $\dagger$. The Journal of Physical Chemistry B, 102(18):3586-3616, apr 1998.

${ }^{75}$ Walter R. P. Scott, Philippe H. Hünenberger, Ilario G. Tironi, Alan E. Mark, Salomon R. Billeter, Jens Fennen, Andrew E. Torda, Thomas Huber, Peter Krüger, and Wilfred F. van Gunsteren. The GROMOS biomolecular simulation program package. The Journal of Physical Chemistry A, 103(19):3596-3607, may 1999. 
${ }^{76}$ David A. Pearlman, David A. Case, James W. Caldwell, Wilson S. Ross, Thomas E. Cheatham, Steve DeBolt, David Ferguson, George Seibel, and Peter Kollman. AMBER, a package of computer programs for applying molecular mechanics, normal mode analysis, molecular dynamics and free energy calculations to simulate the structural and energetic properties of molecules. Computer Physics Communications, 91(1-3):1-41, sep 1995.

${ }^{77}$ Wendy D. Cornell, Piotr Cieplak, Christopher I. Bayly, Ian R. Gould, Kenneth M. Merz, David M. Ferguson, David C. Spellmeyer, Thomas Fox, James W. Caldwell, and Peter A. Kollman. A second generation force field for the simulation of proteins, nucleic acids, and organic molecules. Journal of the American Chemical Society, 117(19):5179-5197, may 1995.

${ }^{78}$ Norman L. Allinger, Young H. Yuh, and Jenn Huei Lii. Molecular mechanics. the MM3 force field for hydrocarbons. 1. Journal of the American Chemical Society, 111(23):8551-8566, nov 1989.

${ }^{79} \mathrm{H}$. Sun. Force field for computation of conformational energies, structures, and vibrational frequencies of aromatic polyesters. Journal of Computational Chemistry, 15(7):752-768, jul 1994.

${ }^{80}$ Huai Sun, Stephen J. Mumby, Jon R. Maple, and Arnold T. Hagler. An ab initio CFF93 all-atom force field for polycarbonates. Journal of the American Chemical Society, 116(7):2978-2987, apr 1994.

${ }^{81} \mathrm{H}$. Sun. COMPASS: an ab initio force-field optimized for condensed-phase ApplicationsOverview with details on alkane and benzene compounds. The Journal of Physical Chemistry B, 102(38):7338-7364, sep 1998.

${ }^{82}$ J. R. Maple, M.-J. Hwang, T. P. Stockfisch, U. Dinur, M. Waldman, C. S. Ewig, and A. T. Hagler. Derivation of class II force fields. i. methodology and quantum force field for the alkyl functional group and alkane molecules. Journal of Computational Chemistry, 15(2):162-182, feb 1994.

${ }^{83}$ Junmei Wang, Romain M. Wolf, James W. Caldwell, Peter A. Kollman, and David A. Case. Development and testing of a general amber force field. Journal of Computational Chemistry, 25(9):11571174, 2004.

${ }^{84}$ S. Lifson, A. T. Hagler, and P. Dauber. Consistent force field studies of intermolecular forces in hydrogen-bonded crystals. 1. carboxylic acids, amides, and the c:o.cntdot..cntdot..cntdot.h- hydrogen bonds. Journal of the American Chemical Society, 101(18):5111-5121, aug 1979.

${ }^{85}$ Pnina Dauber-Osguthorpe, Victoria A. Roberts, David J. Osguthorpe, Jon Wolff, Moniqe Genest, and Arnold T. Hagler. Structure and energetics of ligand binding to proteins:escherichia coli dihydrofolate reductase-trimethoprim, a drug-receptor system. Proteins: Structure, Function, and Genetics, 4(1):31-47, 1988.

${ }^{86}$ Jianhua Liu, Joseph Moo-Young, Matthew McInnis, Melissa A. Pasquinelli, and Lei Zhai. Conjugated polymer assemblies on carbon nanotubes. Macromolecules, 47(2):705-712, jan 2014.

${ }^{87}$ Hendrik Heinz, Tzu-Jen Lin, Ratan Kishore Mishra, and Fateme S. Emami. Thermodynamically consistent force fields for the assembly of inorganic, organic, and biological nanostructures: The INTERFACE force field. Langmuir, 29(6): 1754-1765, jan 2013.

${ }^{88}$ A. K. Rappe, C. J. Casewit, K. S. Colwell, W. A. Goddard, and W. M. Skiff. UFF, a full periodic table force field for molecular mechanics and molecular dynamics simulations. Journal of the American Chemical Society, 114(25):10024-10035, dec 1992.

${ }^{89}$ Stephen L. Mayo, Barry D. Olafson, and William A. Goddard. DREIDING: a generic force field for molecular simulations. The Journal of Physical Chemistry, 94(26):8897-8909, dec 1990.

${ }^{90}$ Syamal S. Tallury and Melissa A. Pasquinelli. Molecular dynamics simulations of polymers with stiff backbones interacting with single-walled carbon nanotubes. The Journal of Physical Chemistry B, 114(29):9349-9355, jul 2010.
${ }^{91}$ Syamal S. Tallury and Melissa A. Pasquinelli. Molecular dynamics simulations of flexible polymer chains wrapping single-walled carbon nanotubes. The Journal of Physical Chemistry B, 114(12):41224129, apr 2010.

${ }^{92}$ FuMing Chen, Wenjing Zhang, Mingli Jia, Li Wei, Xiao-Feng Fan, Jer-Lai Kuo, Yuan Chen, Mary B. Chan-Park, Andong Xia, and Lain-Jong Li. Energy transfer from photo-excited fluorene polymers to single-walled carbon nanotubes. The Journal of Physical Chemistry C, 113(33):14946-14952, jul 2009.

${ }^{93}$ Adri C. T. van Duin, Jan M. A. Baas, and Bastiaan van de Graaf. Delft molecular mechanics: a new approach to hydrocarbon force fields. inclusion of a geometry-dependent charge calculation. Journal of the Chemical Society, Faraday Transactions, 90(19):2881, 1994.

${ }^{94}$ Anthony K. Rappe and William A. Goddard. Charge equilibration for molecular dynamics simulations. The Journal of Physical Chemistry, 95(8):3358-3363, apr 1991.

${ }^{95}$ Jiahao Chen and Todd J. Martínez. QTPIE: Charge transfer with polarization current equalization. a fluctuating charge model with correct asymptotics. Chemical Physics Letters, 438(4-6):315-320, apr 2007.

${ }^{96}$ Adri C. T. van Duin, Siddharth Dasgupta, Francois Lorant, and William A. Goddard. ReaxFF: a reactive force field for hydrocarbons. The Journal of Physical Chemistry A, 105(41):9396-9409, oct 2001.

${ }^{97}$ Yun Kyung Shin, Lili Gai, Sumathy Raman, and Adri C. T. van Duin. Development of a ReaxFF reactive force field for the pt-ni alloy catalyst. The Journal of Physical Chemistry A, 120(41):8044-8055, oct 2016.

${ }^{98}$ Esmaeil Zaminpayma and Kavoos Mirabbaszadeh. Interaction between single-walled carbon nanotubes and polymers: A molecular dynamics simulation study with reactive force field. Computational Materials Science, 58:7-11, jun 2012.

${ }^{99}$ Hasan Metin Aktulga, Sagar A. Pandit, Adri C. T. van Duin, and Ananth Y. Grama. Reactive molecular dynamics: Numerical methods and algorithmic techniques. SIAM Journal on Scientific Computing, 34(1):C1-C23, jan 2012.

${ }^{100}$ H.M. Aktulga, J.C. Fogarty, S.A. Pandit, and A.Y. Grama. Parallel reactive molecular dynamics: Numerical methods and algorithmic techniques. Parallel Computing, 38(4-5):245-259, apr 2012.

${ }^{101}$ Steve Plimpton. Fast parallel algorithms for short-range molecular dynamics. Journal of Computational Physics, 117(1):1-19, mar 1995.

102 https://www.samson-connect.net/.

${ }^{103}$ Xinyang Wang, Qiqiao Lin, Sasikumar Ramachandran, Gaëlle Pembouong, Robert B. Pansu, Isabelle Leray, Bérengère Lebental, and Gaël Zucchi. Optical chemosensors for metal ions in aqueous medium with polyfluorene derivatives: Sensitivity, selectivity and regeneration. Sensors and Actuators B: Chemical, 286:521-532, may 2019.

${ }^{104}$ Kimberly Chenoweth, Adri C. T. van Duin, and William A. Goddard. ReaxFF reactive force field for molecular dynamics simulations of hydrocarbon oxidation. The Journal of Physical Chemistry A, 112(5):1040-1053, feb 2008.

${ }^{105}$ Andrew J. Gellman and Kris R. Paserba. Kinetics and mechanism of oligomer desorption from surfaces: $\mathrm{n}$-alkanes on graphite. The Journal of Physical Chemistry B, 106(51):13231-13241, dec 2002.

${ }^{106}$ Michael Gaus, Albrecht Goez, and Marcus Elstner. Parametrization and benchmark of DFTB3 for organic molecules. Journal of Chemical Theory and Computation, 9(1):338-354, nov 2012.

${ }^{107}$ T. O. Wehling, K. S. Novoselov, S. V. Morozov, E. E. Vdovin, M. I. Katsnelson, A. K. Geim, and A. I. Lichtenstein. Molecular doping of graphene. Nano Letters, 8(1):173-177, jan 2008. 